\title{
THE MORTAR METHOD IN THE WAVELET CONTEXT*
}

\author{
Silvia Bertoluzza ${ }^{1}$ And VAlÉRIE PERrier ${ }^{2}$
}

\begin{abstract}
This paper deals with the use of wavelets in the framework of the Mortar method. We first review in an abstract framework the theory of the mortar method for non conforming domain decomposition, and point out some basic assumptions under which stability and convergence of such method can be proven. We study the application of the mortar method in the biorthogonal wavelet framework. In particular we define suitable multiplier spaces for imposing weak continuity. Unlike in the classical mortar method, such multiplier spaces are not a subset of the space of traces of interior functions, but rather of their duals.

For the resulting method, we provide with an error estimate, which is optimal in the geometrically conforming case.
\end{abstract}

Mathematics Subject Classification. 65N55, 42C40, 65N30, 65N15.

Received: March 30, 2000. Revised: January 23, 2001.

\section{INTRODUCTION}

In the last few years there has been an increasing interest in the use of wavelet based methods for the numerical solution of partial differential equations. The existence of diagonal preconditioners for elliptic operators of any given order [24,28], as well as the possibility of designing efficient adaptive approximation schemes for different type of problems $[9,11,17,22,29]$, are among the attractive features of such bases, which are raising the attention of the scientific computing community on the development in this field.

On the other hand, in order for such methods to be applicable in real life problems, several issues still need to be faced, among which the efficient treatment of non trivial geometries. We recall that such bases were first introduced in $L^{2}(\mathbb{R})$ [31]. Generalizations to $L^{2}(] 0,1[)$ (and to $L^{2}(] 0,1\left[^{d}\right)$ ) have been introduced in the early nineties $[2,19]$, and recently bases were constructed by a conforming domain decomposition approach, allowing to treat all domains which can be split as union of conformal images of rectangles or cubes $[15,26]$.

Rather than following such a philosophy, we prefer to consider here a non conforming domain decomposition approach. This has the great advantage of allowing to couple discretizations of different types. By this approach, wavelet bases can be coupled for instance, with finite elements. This would allow in principle to treat even very complicated geometries, by decomposing any given domain into subdomains, some of which are (conformal images of) squares or cubes - and can then be discretized by wavelets - while the remaining ones can be discretized with finite elements.

Keywords and phrases. Domain decomposition, mortar method, wavelet approximation.

* This work was partially supported by the EC-TMR Network Wavelets in Numerical Simulation, Contract No. ERB-FMRXCT98-0184. It was done partly while the first author was a member of the Laboratoire ASCI (Université Paris Sud, Orsay).

${ }^{1}$ I.A.N.-C.N.R., v. Ferrata 1, 27100, Pavia, Italy. e-mail: aivlis@ian.pv.cnr.it

${ }^{2}$ Laboratoire de Modélisation et Calcul de l'IMAG, BP 53, 38041 Grenoble Cedex 9, France. e-mail: Valerie.Perrier@imag.fr

(c) EDP Sciences, SMAI 2001 
In particular we consider here the mortar method $[7,8]$ which has been designed for such purpose. In the mortar method the interface of the decomposition is itself decomposed into "mortars" (which in this paper will be called "multiplier edges" or "sides"): each "mortar" is a whole edge of a given subdomain, and the "mortars" are disjoint from each other. Weak continuity is imposed by requiring that on each mortar the jump of the approximate solution is orthogonal to a suitable "multiplier space". Such method has nowadays been applied to a wide variety of real-life problems in both two and three dimensions $[3,4,33]$ and it is well suited for parallel implementation [1]. We consider here the second version of such method [5], in which strong continuity of the discrete functions at cross points is not required.

The aim of this paper is to introduce and analyze the application of such a method in the context of biorthogonal wavelet bases. We limit our analysis to the two dimensional case, and for simplicity we assume that all the subdomains are rectangular. For the sake of notational simplicity we chose to split the paper in two parts. In the first part we briefly review the theory of the mortar method. While doing that we underline the basic requirements on the discretization spaces needed in order to get stability and convergence. Moreover we point out the main differences between what happens in the framework of (for instance) finite elements and what will happen when dealing with wavelets.

In the second part we show that, for a suitable choice of the "multiplier space", wavelets fulfill such requirements. Due to the particular structure of the mortar method we are able to do that by concentrating on one subdomain and one "mortar". In particular we provide an error estimate, which, in the case of a geometrically conforming decomposition, is optimal.

\section{THE MORTAR METHOD}

Rather than introducing and analyzing the mortar wavelet method directly, for the sake of notational simplicity we prefer to review at first the theory of the Mortar method in an abstract framework. In doing that, we will point out some basic assumptions, which we will later check in the wavelet case. We follow essentially the guidelines of $[7,8]$. Most of the proofs are the same in an abstract framework as they are in the finite element framework, and we will therefore omit them, or sometimes just briefly sketch them, for the sake of completeness.

On the other hand, when dealing with wavelets, one is naturally confronted with two new issues:

- the natural choice of the multiplier space on an edge is not necessarily a subset of the space of traces of the interior functions, as it is in the classical mortar method; we will see that, under suitable assumptions which are easily verified in the wavelet case, this does not yield any major modification in the results that can be obtained, with no substantial difference with respect to the "classical" mortar method (in this respect see also [36]);

- since interpolation on general non dyadic (even coarse) grids in the wavelet context is still an open problem, we are led to analyze the approximation error also in the case in which the existence of an approximation operator that is interpolating at cross-points is not verified; we will see that this leads (in the geometrically non conforming case) to a loss of a factor of the order of $|\log (h)|$ in the error estimate.

\subsection{The geometry}

Let $\Omega \subset \mathbb{R}^{2}$ be a polygonal domain. We will consider a decomposition of $\Omega$ as the union of $L$ subdomains $\Omega_{\ell}$,

$$
\Omega=\bigcup_{\ell=1}^{L} \Omega_{\ell}
$$

which, for the sake of simplicity we will assume to be rectangular. We set

$$
\begin{aligned}
\Gamma_{\ell n} & =\partial \Omega_{n} \cap \partial \Omega_{\ell} \\
S & =\cup \Gamma_{\ell n} .
\end{aligned}
$$



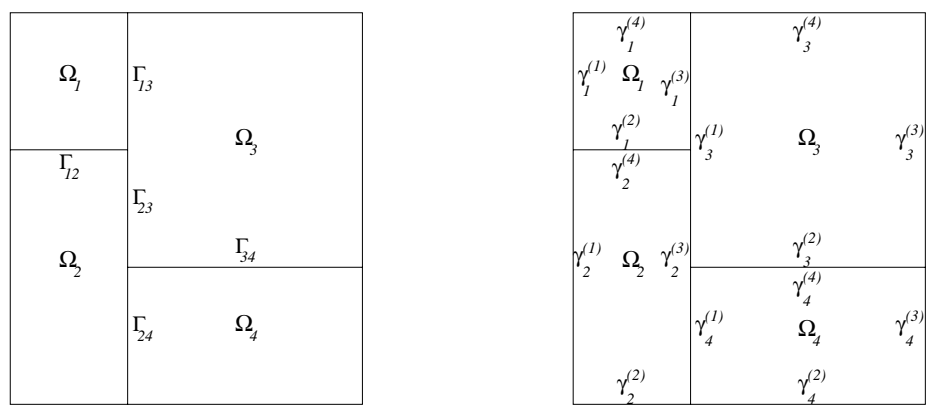

FIGURE 1. Geometrical decomposition of the domain $\Omega$.

Throughout this section we will assume that the above decomposition is fixed. The constants in the bounds that we are going to prove will in general depend on the size of the subdomains, unless otherwise stated.

We denote by $\gamma_{\ell}^{(i)}(i=1, \ldots, 4)$ the $i$-th side of the $\ell$ th subdomain:

$$
\partial \Omega_{\ell}=\bigcup_{i=1}^{4} \gamma_{\ell}^{(i)}
$$

For $\ell=1, \ldots, L$ let $\nu_{\ell}$ denote the outer unit normal to the subdomain $\Omega_{\ell}$, and for $u_{\ell} \in H^{1}\left(\Omega_{\ell}\right)$, let $\partial u_{\ell} / \partial \nu_{\ell}$ denote the outer normal derivative.

Remark 2.1. The decomposition is said to be geometrically conforming if each edge $\gamma_{\ell}^{(i)}$ coincides with $\Gamma_{\ell n}(=$ $\left.\partial \Omega_{\ell} \cap \partial \Omega_{n}\right)$ for some $n$. If the decomposition is not geometrically conforming, then each interior edge $\gamma_{\ell}^{(i)}$ will be in general split as the union of several segments $\Gamma_{\ell n}$ :

$$
\gamma_{\ell}^{(i)}=\bigcup_{n \in I_{\ell}^{(i)}} \Gamma_{\ell n},
$$

where $I_{\ell}^{(i)}$ individuates the set of indices $n$ for which the subdomain $\Omega_{n}$ is adjacent to the edge $\gamma_{\ell}^{(i)}$ of the subdomain $\Omega_{\ell}$ :

$$
I_{\ell}^{(i)}=\left\{n \neq \ell:\left|\partial \Omega_{n} \cap \gamma_{\ell}^{(i)}\right| \neq 0\right\}
$$

We assume that the subdomains are regular in shape, and from now on we will make the following grading assumption (which is automatically fulfilled in the case of a geometrically conforming regular decomposition):

(G1): the following bound holds

$$
\max _{(\ell, i)}\left(\frac{\left|\gamma_{\ell}^{(i)}\right|}{\min _{n \in I_{\ell}^{(i)}}\left|\Gamma_{\ell n}\right|}\right) \leq \rho .
$$

The constants appearing in the estimates of the following sections will in general depend on the bound $\rho$.

For defining the mortar method we start by choosing a splitting of the skeleton $S$ as the disjoint union of a certain number of subdomain sides $\gamma_{\ell}^{(i)}$, which we will call "multiplier sides" (in the usual terminology these are called "non mortars" or "slave sides"). More precisely, we choose an index set $I \subset\{1, \ldots, L\} \times\{1, \ldots, 4\}$ 

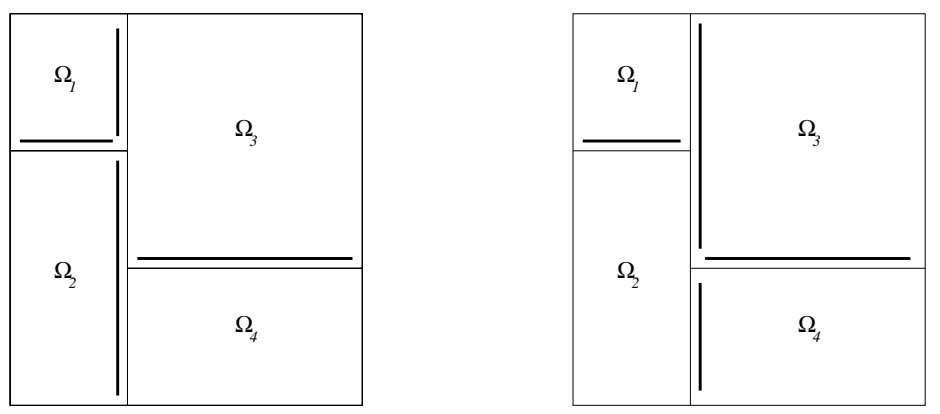

Figure 2. Two possible choices of "multiplier sides". For the one on the left we have (using the indexing of Fig. 1) $I=\{(2,3),(1,2),(1,3),(3,2)\}$ and $I^{*}=\{(4,1),(4,4),(3,1),(2,4)\}$ while for the one on the right $I=\{(1,2),(3,1),(3,2),(4,1)\}$. Remark that in all cases the set $\{1, \ldots, L\} \times\{1, \ldots, 4\} \backslash I \cup I^{*}$ individuates the edges which belong to the external boundary $\partial \Omega$.

such that

$$
S=\bigcup_{(\ell, i) \in I} \gamma_{\ell}^{(i)}, \quad \begin{gathered}
\left(\ell_{1}, i_{1}\right),\left(\ell_{2}, i_{2}\right) \in I, \\
\left(\ell_{1}, i_{1}\right) \neq\left(\ell_{2}, i_{2}\right)
\end{gathered} \Rightarrow \gamma_{\ell_{1}}^{\left(i_{1}\right)} \cap \gamma_{\ell_{2}}^{\left(i_{2}\right)}=\emptyset
$$

Furthermore we will denote by $I^{*} \subset\{1, \cdots, L\} \times\{1, \cdots, 4\}$ the index-set corresponding to "trace sides" ("mortars" or "master sides" in the usual terminology), which is defined in such a way that $I^{*} \cap I=\emptyset$ and $S=\cup_{(\ell, i) \in I^{*}} \gamma_{\ell}^{(i)}$.

Corresponding to the splitting (7) we define a normal direction on the skeleton. More precisely for $(\ell, i) \in I$ $\left(\gamma_{\ell}^{(i)}\right.$ multiplier side) we set

$$
\nu=\nu_{\ell} \quad \text { on } \gamma_{\ell}^{(i)}
$$

Since $S=\cup_{(\ell, i) \in I} \gamma_{\ell}^{(i)}$, this defines $\nu$ on $S$. For any $u=\left(u_{\ell}\right)_{\ell=1, \ldots, L} \in \prod_{\ell=1}^{L} H^{1}\left(\Omega_{\ell}\right)$, $\frac{\partial u}{\partial \nu}$ will be defined accordingly: on $\gamma_{\ell}^{(i)},(\ell, i) \in I\left(\gamma_{\ell}^{(i)}\right.$ multiplier side) we set $\frac{\partial u}{\partial \nu}=\frac{\partial u_{\ell}}{\partial \nu_{\ell}}$. Moreover, we let $[u]$ denote the jump of $u$ along the skeleton $S$ with the proper sign: on $\Gamma_{\ell n} \subset \gamma_{\ell}^{(i)}$, with $(\ell, i) \in I\left(\gamma_{\ell}^{(i)}\right.$ multiplier side),

$$
\left.[u]\right|_{\Gamma_{\ell n}}=\left.u_{\ell}\right|_{\Gamma_{\ell n}}-\left.u_{n}\right|_{\Gamma_{\ell n}}
$$

In order to simplify the notation in the following we will sometimes make use of a multi-index $m=(\ell, i)$. We will for instance write $\gamma_{m}, m \in I$ (respectively $m \in I^{*}$ ) for $\gamma_{\ell}^{(i)},(\ell, i) \in I$ (respectively $(\ell, i) \in I^{*}$ ).

In the following, letting $\hat{\Omega}$ be either one of the domains $\Omega, \Omega_{\ell}, \partial \Omega_{\ell}, \gamma_{\ell}^{(i)}$ and $\Gamma_{\ell n}$, we will denote by $\|\cdot\|_{s, \hat{\Omega}}$ (resp. $|\cdot|_{s, \hat{\Omega}}$ ) the norm (resp. seminorm) of the space $H^{s}(\hat{\Omega})$. Moreover, we will also make use of the norm of the space $H_{00}^{1 / 2}(\hat{\Omega})$, which we will simply denote by $\|\cdot\|_{H_{00}^{1 / 2}(\hat{\Omega})}$. 


\subsection{The continuous problem}

For simplicity we will consider the following model problem. Given $f \in L^{2}(\Omega)$, find $u: \Omega \longrightarrow \mathbb{R}$ such that

$$
\begin{cases}-\Delta u=f, & \text { in } \Omega, \\ u=0, & \text { on } \partial \Omega .\end{cases}
$$

Given the splitting of the domain $\Omega$ introduced in the previous section, we will consider a non conforming domain decomposition method for the solution of such a problem. In order to do that let

$$
\begin{gathered}
X=\prod_{\ell=1}^{L}\left\{u_{\ell} \in H^{1}\left(\Omega_{\ell}\right) \mid u_{\ell}=0 \text { on } \partial \Omega \cap \partial \Omega_{\ell}\right\}, \\
T=\prod_{\ell=1}^{L} H_{*}^{1 / 2}\left(\partial \Omega_{\ell}\right),
\end{gathered}
$$

where $H_{*}^{1 / 2}\left(\partial \Omega_{\ell}\right)$ is defined by

$$
H_{*}^{1 / 2}\left(\partial \Omega_{\ell}\right)=H^{1 / 2}\left(\partial \Omega_{\ell}\right) \quad \text { if } \partial \Omega_{\ell} \cap \partial \Omega=\emptyset
$$

and

$$
H_{*}^{1 / 2}\left(\partial \Omega_{\ell}\right)=\left\{\eta \in H^{1 / 2}\left(\partial \Omega_{\ell}\right),\left.\eta\right|_{\partial \Omega_{\ell} \cap \partial \Omega} \equiv 0\right\} \sim H_{00}^{1 / 2}\left(\partial \Omega_{\ell} \backslash \partial \Omega\right),
$$

otherwise. The space $H_{*}^{1 / 2}\left(\partial \Omega_{\ell}\right)$ will be endowed with the norm $\|\cdot\|_{1 / 2, \partial \Omega_{\ell}}$, and we will denote by $\|\cdot\|_{-1 / 2, \ell}$ the norm of the corresponding dual space. Remark that in general $\|\cdot\|_{-1 / 2, \ell} \neq\|\cdot\|_{-1 / 2, \partial \Omega_{\ell}}$.

Remark 2.2. Remark that by definition the elements of both $X$ and $T$ (and in the sequel the elements of the discrete subspaces $X_{\delta}$ and $T_{\delta}$ ) satisfy an homogeneous boundary condition on $\partial \Omega$.

On $X$ we introduce the following broken norm and semi-norm:

$$
\|u\|_{X}=\left(\sum_{\ell=1}^{L}\|u\|_{1, \Omega_{\ell}}^{2}\right)^{\frac{1}{2}}, \quad|u|_{X}=\left(\sum_{\ell=1}^{L}|u|_{1, \Omega_{\ell}}^{2}\right)^{\frac{1}{2}} .
$$

In the following it will also be convenient to introduce the following norm on $T$ :

$$
\|\eta\|_{T}=\left(\sum_{\ell=1}^{L}\left\|\eta_{\ell}\right\|_{1 / 2, \partial \Omega_{\ell}}^{2}\right)^{1 / 2} .
$$

Moreover, for $\lambda \in L^{2}(S)$ we will use the notation:

$$
\|\lambda\|_{-1 / 2, S}=\left(\sum_{\ell=1}^{L}\|\lambda\|_{-1 / 2, \ell}^{2}\right)^{1 / 2} .
$$

Let now a composite bilinear form $a_{X}: X \times X \longrightarrow \mathbb{R}$ be defined as follows:

$$
a_{X}(u, v)=\sum_{\ell=1}^{L} \int_{\Omega_{\ell}} \nabla u_{\ell} \nabla v_{\ell}
$$


The bilinear form $a_{X}$ is clearly not coercive on $X$. In order to obtain a well posed problem we will then consider proper subspaces of $X$, consisting of functions satisfying a suitable weak continuity constraint.

More precisely, for any subspace $M$ of $L^{2}(S)$ let a constrained space $\mathcal{X}(M)$ be defined as follows:

$$
\mathcal{X}(M)=\left\{u \in X: \int_{S}[u] \lambda=0, \forall \lambda \in M\right\} .
$$

We will consider the following problem (depending on the choice of the "multiplier space" $M$ ):

Problem 2.3 $\left(P_{M}\right)$. Find $u_{M} \in \mathcal{X}(M)$ such that for all $v \in \mathcal{X}(M)$

$$
a_{X}\left(u_{M}, v\right)=\int_{\Omega} f v
$$

In the following we will consider multiplier spaces $M$ satisfying the following assumption.

(BP): There exists a constant $C_{M}>0$ such that the following broken Poincaré inequality holds for all $u \in$ $\mathcal{X}(M)$ :

$$
|u|_{X} \geq C_{M}\|u\|_{X}
$$

The bound (19) is evidently equivalent to the coercivity of the bilinear form $a_{X}$ over $\mathcal{X}(M)$. By simply observing that $M_{1} \subset M_{2}$ implies $\mathcal{X}\left(M_{2}\right) \subset \mathcal{X}\left(M_{1}\right)$ one gets the following well known result (see for instance [7]).

Theorem 2.4. Let $\bar{M}$ satisfy assumption (BP). Then for all $M$ such that $\bar{M} \subset M$ we have the following:

- the solution $u_{M}$ of problem $P_{M}$ exists and is unique;

- for $u$ solution of (10) the following bound holds with constant $\bar{C}=\bar{C}(\bar{M})$ :

$$
\left\|u-u_{M}\right\|_{X} \leq \bar{C} \inf _{\lambda \in M}\left\|\frac{\partial u}{\partial \nu}-\lambda\right\|_{-1 / 2, S}
$$

\subsection{Mortar discrete problem - Approximation spaces}

For each $\ell$ let now $\mathcal{V}_{\delta}^{\ell}$ be a family of finite dimensional subspaces of $H^{1}\left(\Omega_{\ell}\right) \cap C^{0}\left(\bar{\Omega}_{\ell}\right)$, depending on a parameter $\delta=\delta_{\ell}>0$ and satisfying an homogeneous boundary condition on $\partial \Omega \cap \partial \Omega_{\ell}$. Set

$$
T_{\delta}^{\ell}=\left.\mathcal{V}_{\delta}^{\ell}\right|_{\partial \Omega_{\ell}}
$$

and, for each edge $\gamma_{\ell}^{(i)}$ of the subdomain $\Omega_{\ell}$ let

$$
\begin{aligned}
& T_{\ell, i}=\left\{\eta: \eta \text { is the trace on } \gamma_{\ell}^{(i)} \text { of some } u_{\ell} \in \mathcal{V}_{\delta}^{\ell}\right\} \\
& T_{\ell, i}^{0}=\left\{\eta \in T_{\ell, i}: \eta=0 \text { at the vertices of } \gamma_{\ell}^{(i)}\right\} .
\end{aligned}
$$

We set

$$
X_{\delta}=\prod_{\ell=1}^{L} \mathcal{V}_{\delta}^{\ell} \subset X, \quad T_{\delta}=\prod_{\ell=1}^{L} T_{\delta}^{\ell} \subset T
$$

For each $m=(\ell, i) \in I$ let a finite dimensional multiplier space $M_{\delta}^{m}$ on $\gamma_{m}$ be given, also depending on the parameter $\delta$ :

$$
M_{\delta}^{m} \subset L^{2}\left(\gamma_{m}\right), \quad \quad \operatorname{dim}\left(M_{\delta}^{m}\right)=\operatorname{dim}\left(T_{m}^{0}\right)
$$


We set:

$$
M_{\delta}=\left\{\eta \in L^{2}(S),\left.\eta\right|_{\gamma_{m}} \in M_{\delta}^{m} \forall m \in I\right\} \sim \prod_{m \in I} M_{\delta}^{m}
$$

The constrained approximation and trace spaces $\mathcal{X}_{\delta}$ and $\mathcal{T}_{\delta}$ are then defined as follows:

$$
\begin{aligned}
& \mathcal{X}_{\delta}=\left\{v_{\delta} \in X_{\delta}, \int_{S}\left[v_{\delta}\right] \lambda=0, \forall \lambda \in M_{\delta}\right\} \subset \mathcal{X}\left(M_{\delta}\right), \\
& \mathcal{T}_{\delta}=\left\{\eta \in T_{\delta}, \int_{S}[\eta] \lambda=0, \forall \lambda \in M_{\delta}\right\} .
\end{aligned}
$$

We can now introduce the following discrete problem:

Problem 2.5 (PD). Find $u_{\delta} \in \mathcal{X}_{\delta}$ such that for all $v_{\delta} \in \mathcal{X}_{\delta}$

$$
a_{X}\left(u_{\delta}, v_{\delta}\right)=\int_{\Omega} f v_{\delta}
$$

The following result holds:

Theorem 2.6. Assume that $\bar{M}=\cap_{\delta>0} M_{\delta}$ satisfies assumption (BP). Then for all $\delta>0$, problem $P_{\delta}$ admits a unique solution $u_{\delta}$ which satisfies the following error estimate:

$$
\left\|u-u_{\delta}\right\|_{X} \leq \bar{C}\left(\inf _{v_{\delta} \in \mathcal{X}_{\delta}}\left\|u-v_{\delta}\right\|_{X}+\inf _{\lambda \in M_{\delta}}\left\|\frac{\partial u}{\partial \nu}-\lambda\right\|_{-1 / 2, S}\right)
$$

with $\bar{C}$ constant depending on $\bar{M}$.

\subsection{Stability}

In order to apply the result of the previous sections, we need to choose the multiplier spaces $M_{\delta}^{m}$ in such a way that $\bar{M}=\cap_{\delta} M_{\delta} \sim \prod_{m \in I} \cap_{\delta} M_{\delta}^{m}$ satisfies assumption (BP). We would like to recall that assumption (BP) is much less restrictive than it might seem at first sight. It is well known [7] that in the framework considered here, a sufficient condition for (BP) to hold is the following: for all $m=(\ell, i) \in I$ ( $\gamma_{\ell}^{(i)}$ multiplier side), for any piecewise constant function $g, g$ constant on each $\Gamma_{\ell n}, n \in I_{\ell}^{(i)}$ (we recall that $\gamma_{m}=\bigcup_{n \in I_{\ell}^{(i)}} \Gamma_{\ell n}$ ), we have

$$
\int_{\gamma_{m}} g \lambda=0, \forall \lambda \in \bar{M}_{m}=\cap_{\delta} M_{\delta}^{m} \quad \text { implies } \quad g=0 .
$$

In fact, roughly speaking, any function for which $|\cdot|_{X}=0$, does necessarily take a constant value on each subdomain, and, if it belongs to the constrained space $\mathcal{X}(\bar{M})$, by (31) such constant values agree. Since the functions in $\mathcal{X}(\bar{M})$ vanish at the boundary, the function is then identically zero. The validity of assumption (31) has been studied for several types of discretizations. Several sufficient conditions for it to hold are therefore known. We recall for instance the following result [6] which can be applied in our framework.

Proposition 2.7 (Sufficient condition I). If $\operatorname{card}\left\{I_{\ell}^{(i)}\right\} \leq \tilde{N}$ (card $\left\{I_{\ell}^{(i)}\right\}$ being the number of subdomains adjacent to $\gamma_{\ell}^{(i)}$ and $\tilde{N}$ being the number of polynomials exactly reproduced in $\bar{M}_{m}$ ), then (31) holds. 
It is also easy to prove that the following condition holds.

Proposition 2.8 (Sufficient condition II). A sufficient condition for $\bar{M}_{m}$ to satisfy (31) is that $\forall n \in I_{\ell}^{(i)}$ there $\exists \beta_{n} \in \bar{M}_{m}$ such that

$$
\operatorname{supp} \beta_{n} \subset \Gamma_{\ell n}, \quad \int_{\Gamma_{\ell n}} \beta_{n}=1 .
$$

In fact, for $g=c_{n}$ on $\Gamma_{\ell n}$ we have $\int_{\gamma_{m}} g \beta_{n}=\int_{\Gamma_{\ell n}} g \beta_{n}=c_{n}$, and if $g$ is orthogonal to all the functions in $\bar{M}_{m}$ this implies $c_{n}=0$.

Remark 2.9. In the case of a geometrically conforming decomposition (for all $m=(\ell, k), \gamma_{m}=\Gamma_{\ell n}$, for some $n$ ) the above condition is also necessary, which is in general not true in the case of geometrically non conforming decompositions (see for instance the case of the mortar element method in the framework of a spectral decomposition, in which case the elements of $M_{\delta}^{m}$ are polynomial functions).

\subsection{Approximation error}

In order to bound the right hand side of (30) we will make the following assumptions on the spaces considered:

(A1): $\forall m=(\ell, i) \in I\left(\gamma_{\ell}^{(i)}\right.$ multiplier side), there exists a bounded projection operator $\pi_{m}: L^{2}\left(\gamma_{m}\right) \longrightarrow T_{m}^{0}$, such that for all $\eta \in L^{2}\left(\gamma_{m}\right)$ and for all $\lambda \in M_{\delta}^{m}$

$$
\int_{\gamma_{m}}\left(\eta-\pi_{m} \eta\right) \lambda=0
$$

and for all $\eta \in H_{00}^{1 / 2}\left(\gamma_{m}\right)$

$$
\left\|\pi_{m} \eta\right\|_{H_{00}^{1 / 2}\left(\gamma_{m}\right)} \lesssim\|\eta\|_{H_{00}^{1 / 2}\left(\gamma_{m}\right)}
$$

(A2): $\forall m=(\ell, i) \in I\left(\gamma_{\ell}^{(i)}\right.$ multiplier side), there exists a discrete lifting $R_{m}: T_{m}^{0} \longrightarrow \mathcal{V}_{\delta}^{\ell}$ such that for all $\eta \in T_{m}^{0}$

$$
R_{m} \eta=0 \text { on } \partial \Omega_{\ell} \backslash \gamma_{m}, \quad R_{m} \eta=\eta \text { on } \gamma_{m},
$$

and

$$
\left\|R_{m} \eta\right\|_{1, \Omega_{\ell}} \lesssim\|\eta\|_{H_{00}^{1 / 2}\left(\gamma_{m}\right)}
$$

(A3): for all $m=(\ell, i) \in I$ ( $\gamma_{\ell}^{(i)}$ multiplier side), the following inverse inequality holds: for all elements $\eta \in T_{m}^{0}$ and for all $s, 0 \leq s<1 / 2$ it holds

$$
\|\eta\|_{H_{00}^{1 / 2}\left(\gamma_{m}\right)} \lesssim h_{m}^{s-1 / 2}\|\eta\|_{s, \gamma_{m}},
$$

where $h_{m}$ is a discretization parameter acting as "mesh size" on $\gamma_{m}$.

Remark 2.10. It is well known [35] that for $s<1 / 2$ the Sobolev space $H^{s}(G)$ ( $G$ bounded domain) can be obtained by space interpolation both as $\left[L^{2}(G), H^{1}(G)\right]_{s}$ and as $\left[L^{2}(G), H_{0}^{1}(G)\right]_{s}$, the two resulting norms being equivalent. However, since the constants in the norm equivalence explode as $s$ tends to $1 / 2$, it is not difficult to realize that for (37) to hold uniformly in $s, H^{s}\left(\gamma_{m}\right)$ has to be defined as $\left[L^{2}\left(\gamma_{m}\right), H_{0}^{1}\left(\gamma_{m}\right)\right]_{s}$. In fact, such inverse inequalities are usually proven for $s=0$ and then extended to $s \in(0,1 / 2)$ by space interpolation. If 
then $H^{s}(G)$ is defined as $\left[L^{2}(G), H_{0}^{1}(G)\right]_{s}$, it is possible to prove that for $G=\cup_{n} G_{n}, G$ and $G_{n}$ intervals, it holds for all $u \in H^{1 / 2-\varepsilon}(G)$

$$
\sum_{n}\|u\|_{1 / 2-\varepsilon, G_{n}}^{2} \lesssim \frac{1}{\varepsilon^{2}}\|u\|_{1 / 2-\varepsilon, G}^{2} .
$$

Moreover we remark that, also by space interpolation, assumption (A1) implies that the projection operator $\pi_{m}$ verifies for all $s, 0<s<1 / 2$ :

$$
\left\|\pi_{m} \eta\right\|_{s, \gamma_{m}} \lesssim\|\eta\|_{s, \gamma_{m}}
$$

uniformly in $s$.

Letting

$$
h=\min _{m \in I} h_{m}
$$

the following lemma holds:

Lemma 2.11. If assumptions (A1-A3) hold, then for any $\eta=\left(\eta_{\ell}\right)_{\ell=1, \ldots, L} \in T$ it holds:

$$
\sum_{m \in I}\left\|\pi_{m}([\eta])\right\|_{H_{00}^{1 / 2}\left(\gamma_{m}\right)}^{2} \lesssim(1+|\log h|)^{2}\|\eta\|_{T}^{2} .
$$

Proof. Let $\eta=\left(\eta_{\ell}\right)_{\ell=1, \ldots, L}$ be any element of $T=\prod_{\ell=1, \ldots, L} H_{*}^{1 / 2}\left(\partial \Omega_{\ell}\right)$. Using (37) and (39), for any $\varepsilon$, $0<\varepsilon<1 / 2$ it holds:

$$
\begin{aligned}
\sum_{m \in I}\left\|\pi_{m}([\eta])\right\|_{H_{00}^{1 / 2}\left(\gamma_{m}\right)}^{2} & \lesssim \sum_{m \in I} h_{m}^{-2 \varepsilon}\left\|\pi_{m}([\eta])\right\|_{1 / 2-\varepsilon, \gamma_{m}}^{2} \\
& \lesssim \sum_{\substack{m \in I \\
m=(\ell, i)}} h_{m}^{-2 \varepsilon} \sum_{n \in I_{\ell}^{(i)}}\left(\left\|\eta_{\ell}\right\|_{1 / 2-\varepsilon, \Gamma_{\ell n}}^{2}+\left\|\eta_{n}\right\|_{1 / 2-\varepsilon, \Gamma_{\ell n}}^{2}\right) \\
& =\sum_{\ell=1}^{L} h^{-2 \varepsilon} \sum_{n: \Gamma_{\ell n} \neq \emptyset}\left\|\eta_{\ell}\right\|_{1 / 2-\varepsilon, \Gamma_{\ell n}}^{2} \lesssim \frac{h^{-2 \varepsilon}}{\varepsilon^{2}} \sum_{\ell=1}^{L}\left\|\eta_{\ell}\right\|_{1 / 2, \partial \Omega_{\ell}}^{2}
\end{aligned}
$$

where the last bound derives from (38). Choosing $\varepsilon=\frac{1}{|\log h|}$ we get (40).

We can now define a linear operator $\pi: \prod_{\ell=1}^{L} L^{2}\left(\partial \Omega_{\ell}\right) \longrightarrow \prod_{\ell=1}^{L} L^{2}\left(\partial \Omega_{\ell}\right)$ that we will need in the following: more precisely, for $\eta=\left(\eta_{\ell}\right)_{\ell=1, \ldots, L}, \pi(\eta)$ is defined on multiplier sides as $\pi_{m}$ applied to the jump of $\eta$, while it is set identically zero on trace sides and on the external boundary $\partial \Omega$ :

$$
\begin{aligned}
\pi(\eta)=\left(\eta_{\ell}^{*}\right)_{\ell=1, \ldots, L}, \text { with } & \\
\left.\eta_{\ell}^{*}\right|_{\gamma_{m}} & =\pi_{m}\left(\left.[\eta]\right|_{\gamma_{m}}\right), \text { for } m=(\ell, i) \in I \\
\left.\eta_{\ell}^{*}\right|_{\gamma_{m}} & =0, \text { for } m=(\ell, i) \in I^{*}, \\
\eta_{\ell}^{*} & \equiv 0 \text { on } \partial \Omega_{\ell} \cap \partial \Omega .
\end{aligned}
$$


By observing that for all $\eta \in H^{1 / 2}\left(\partial \Omega_{\ell}\right)$ satisfying $\left.\eta\right|_{\gamma_{\ell}^{(i)}} \in H_{00}^{1 / 2}\left(\gamma_{\ell}^{(i)}\right),(i=1, \ldots, 4)$ it holds

$$
\|\eta\|_{1 / 2, \partial \Omega_{\ell}}^{2} \lesssim \sum_{i=1}^{4}\|\eta\|_{H_{00}^{1 / 2}\left(\gamma_{\ell}^{(i)}\right)}^{2}
$$

we obtain the following corollary of Lemma 2.11 .

Corollary 2.12. If assumptions (A1-A3) hold, then for any $\eta=\left(\eta_{\ell}\right)_{\ell=1, \ldots, L}$ in the trace space $T$ it holds:

$$
\|\pi(\eta)\|_{T} \lesssim(1+|\log h|)\|\eta\|_{T}
$$

We are then able to prove the following theorem.

Theorem 2.13. Let assumptions (A1-A3) hold. Then for any $u \in H_{0}^{1}(\Omega)$ we have:

$$
\inf _{v_{\delta} \in \mathcal{X}_{\delta}}\left\|u-v_{\delta}\right\|_{X} \lesssim(1+|\log h|)\left(\sum_{\ell=1}^{L} \inf _{v_{\delta, \ell} \in \mathcal{V}_{\delta}^{\ell}}\left\|u-v_{\delta, \ell}\right\|_{1, \Omega_{\ell}}^{2}\right)^{1 / 2} .
$$

Proof. For each $\ell$ let $w_{\delta, \ell}$ be an arbitrary element of $\mathcal{V}_{\delta}^{\ell}$. Let $\bar{w}_{\delta}=\left(w_{\delta, \ell}\right)_{\ell=1, \ldots, L}$. Since $\bar{w}_{\delta}$ does not necessarily satisfy the jump condition, it may not belong to $\mathcal{X}_{\delta}$. We now define an element $w_{\delta} \in \mathcal{X}_{\delta}$ as follows:

$$
w_{\delta}=\bar{w}_{\delta}-\sum_{m \in I} R_{m} \pi_{m}\left(\left[\bar{w}_{\delta}\right]\right),
$$

where, by abuse of notation for $\eta \in T_{m}^{0}(m=(\ell, i))$ we indicate by $R_{m}(\eta)$ the element of $\mathcal{X}_{\delta}$ which coincides with $R_{m}(\eta)$ in $\Omega_{\ell}$ and which is identically zero on the other subdomains. We easily check that $w_{\delta}$ belongs to $\mathcal{X}_{\delta}$. In fact for $\eta \in T_{m}^{0}$ we have,

$$
\left[R_{m} \eta\right]=\eta \text { on } \gamma_{m},
$$

and hence

$$
\left[w_{\delta}\right]=\left[\bar{w}_{\delta}\right]-\pi_{m}\left(\left[\bar{w}_{\delta}\right]\right) \text { on } \gamma_{m},
$$

which implies, for $\lambda_{\delta} \in M_{\delta}$, thanks to (33),

$$
\int_{S}\left[w_{\delta}\right] \lambda_{\delta}=\sum_{m \in I} \int_{\gamma_{m}}\left(\left[\bar{w}_{\delta}\right]-\pi_{m}\left(\left[\bar{w}_{\delta}\right]\right)\right) \lambda_{\delta}=0 .
$$

We can now bound

$$
\begin{aligned}
\left\|\sum_{m \in I} R_{m} \pi_{m}\left(\left[\bar{w}_{\delta}\right]\right)\right\|_{X}^{2} & =\sum_{\ell=1}^{L}\left\|\sum_{\substack{m \in I \\
m=\ell, i)}} R_{m} \pi_{m}\left(\left[\bar{w}_{\delta}\right]\right)\right\|_{1, \Omega_{\ell}}^{2} \lesssim \sum_{\ell=1}^{L} \sum_{\substack{m \in I \\
m=(\ell, i)}}\left\|R_{m} \pi_{m}\left(\left[\bar{w}_{\delta}\right]\right)\right\|_{1, \Omega_{\ell}}^{2} \\
& \lesssim \sum_{m \in I}\left\|\pi_{m}\left(\left[\bar{w}_{\delta}\right]\right)\right\|_{H_{00}^{1 / 2}\left(\gamma_{m}\right)}^{2}=\sum_{m \in I}\left\|\pi_{m}\left(\left[\bar{w}_{\delta}-u\right]\right)\right\|_{H_{00}^{1 / 2}\left(\gamma_{m}\right)}^{2},
\end{aligned}
$$


where the last equality descends from the observation that for $u \in H^{1}(\Omega)$ it holds $[u]=0$, and hence $\left[\bar{w}_{\delta}\right]=$ $\left[\bar{w}_{\delta}-u\right]$. Thus, by applying lemma 2.11 for $\eta=\left[\bar{w}_{\delta}-u\right]$, using a classical trace theorem we obtain

$$
\begin{aligned}
\inf _{v_{\delta} \in \mathcal{X}_{\delta}}\left\|u-v_{\delta}\right\|_{X}^{2} & \lesssim\left\|u-\bar{w}_{\delta}\right\|_{X}^{2}+\left\|\sum_{m \in I} R_{m} \pi_{m}\left(\left[\bar{w}_{\delta}\right]\right)\right\|_{X}^{2} \\
& \lesssim(1+|\log h|)^{2} \sum_{\ell=1}^{L}\left\|u-w_{\delta, \ell}\right\|_{1, \Omega_{\ell}}^{2} .
\end{aligned}
$$

Since the $w_{\delta, \ell}$ are arbitrary, we get the thesis.

Corollary 2.14. Let the family $\mathcal{V}_{\delta}^{\ell}$ be given and let $M_{\delta}$ be defined by (26) and $\mathcal{X}_{\delta}$ by (27). Assume that the following properties hold:

(i) accuracy: assumptions (A1-A3) are satisfied for $T_{m}^{0}$ and $M_{\delta}^{m}$ defined by (23), (25);

(ii) stability: $\bar{M}=\cap_{\delta} M_{\delta}$ satisfies assumption (BP).

Then, for $u$ solution of (10) and $u_{\delta}$ solution of (29) the following error estimate holds:

$$
\begin{aligned}
\left\|u-u_{\delta}\right\|_{X} \lesssim & (1+|\log h|)\left(\sum_{\ell=1}^{L} \inf _{v_{\delta, \ell} \in \mathcal{V}_{\delta}^{\ell}}\left\|u-v_{\delta, \ell}\right\|_{1, \Omega_{\ell}}^{2}\right)^{1 / 2} \\
& +\inf _{\lambda \in M_{\delta}}\left\|\frac{\partial u}{\partial \nu}-\lambda\right\|_{-1 / 2, S} .
\end{aligned}
$$

Remark 2.15. We remark that assumptions (A1-A3) deal independently with each subdomain and also with the coupling between the discretization on a subdomain and the multiplier space $M_{\delta}^{m}$ defined on each one of its sides. In the construction of suitable discretization spaces, it will then be sufficient to study the properties of the discretization on one subdomain and the multiplier space induced on one of its sides.

Clearly, Theorem 2.13 yields only a sub-optimal error estimate, where, due to the constraint, a factor of the order $|\log h|$ is lost with respect to the optimal approximation rate. Nevertheless, if the solution is sufficiently regular, an optimal error estimate can be retrieved, provided that a suitable projector exists, verifying an interpolation property at cross points. More precisely the following theorem holds.

Theorem 2.16. For some $\bar{s}>1$, assume that $\mathcal{V}_{\delta}^{\ell} \subset H^{\bar{s}}\left(\Omega_{\ell}\right)$ and that operators $\Pi_{\delta, \ell}: H^{\bar{s}}\left(\Omega_{\ell}\right) \rightarrow \mathcal{V}_{\delta}^{\ell}$ exist such that

- $\forall s, t, 0 \leq s \leq \bar{s}, \bar{s} \leq t \leq \bar{t}$, (t possibly depending on $\ell$ ) for all $u \in H^{t}\left(\Omega_{\ell}\right)$ with $u=0$ on $\partial \Omega_{\ell} \cap \partial \Omega$ we have

$$
\left\|u-\Pi_{\delta, \ell} u\right\|_{s, \Omega_{\ell}} \lesssim \delta_{\ell}^{t-s}\|u\|_{t, \Omega_{\ell}},
$$

$\delta_{\ell}$ being the "mesh-size" of the discretization in the subdomain $\Omega_{\ell}$;

- for all $A \in \partial \Omega_{\ell}$ such that $A$ is a vertex of $\Gamma_{\ell n}$ for some $n$,

$$
\Pi_{\delta, \ell} u(A)=u(A), \quad \forall u \in H^{\bar{s}}\left(\Omega_{\ell}\right) .
$$

Then if $u \in H^{\bar{s}}(\Omega) \cap H_{0}^{1}(\Omega)$ satisfies $\left.u\right|_{\Omega_{\ell}} \in H^{t_{\ell}}\left(\Omega_{\ell}\right)\left(\bar{s} \leq t_{\ell} \leq \bar{t}\right)$ it holds

$$
\inf _{v_{\delta} \in \mathcal{X}_{\delta}}\left\|u-v_{\delta}\right\|_{X} \lesssim \sum_{\ell=1}^{L} \delta_{\ell}^{t_{\ell}-1}\|u\|_{t_{\ell}, \Omega_{\ell}} .
$$

Remark 2.17. We point out that in the case of general discretizations, for $m=(\ell, i) \in I$ ( $\gamma_{\ell}^{(i)}$ multiplier side) the two mesh size parameters $h_{m}$ in the inverse inequality (37) (corresponding to the "finest mesh size" on $\gamma_{m}$ ) and $\delta_{\ell}$ in the direct inequality (48) (corresponding to the "coarsest mesh size" in $\Omega_{\ell}$ ) do not necessarily coincide. 
Proof. Let $w_{\delta, \ell}=\Pi_{\delta, \ell} u$. Setting $\bar{w}_{\delta}=\left(w_{\delta, \ell}\right)_{\ell=1, \ldots, L}$ and $w_{\delta}=\bar{w}_{\delta}-\sum_{m \in I} R_{m} \pi_{m}\left(\left[\bar{w}_{\delta}\right]\right)$, as in the proof of Theorem 2.13 we have

$$
\inf _{v_{\delta} \in \mathcal{X}_{\delta}}\left\|u-v_{\delta}\right\|_{X} \lesssim\left\|u-w_{\delta}\right\|_{X} \lesssim \sum_{\ell=1}^{L}\left\|u-\Pi_{\delta, \ell} u\right\|_{1, \Omega_{\ell}}+\sum_{m \in I}\left\|\pi_{m}\left(\left[\bar{w}_{\delta}-u\right]\right)\right\|_{H_{00}^{1 / 2}\left(\gamma_{m}\right)} .
$$

Thanks to (49), $\left[w_{\delta}-u\right]$ belongs to $H_{00}^{1 / 2}\left(\gamma_{m}\right)$, hence we can write

$$
\sum_{m \in I}\left\|\pi_{m}\left(\left[\bar{w}_{\delta}-u\right]\right)\right\|_{H_{00}^{1 / 2}\left(\gamma_{m}\right)} \lesssim \sum_{m \in I}\left\|\left[\bar{w}_{\delta}-u\right]\right\|_{H_{00}^{1 / 2}\left(\gamma_{m}\right)} .
$$

We now observe that $\left.\left[\bar{w}_{\delta}-u\right]\right|_{\Gamma_{\ell n}} \in H_{00}^{1 / 2}\left(\Gamma_{\ell n}\right)$ for all $\ell, n$. Then, by writing it as the sum of functions $\zeta^{n}$ each one coinciding with $\left[\bar{w}_{\delta}-u\right]$ on $\Gamma_{\ell n}$ and vanishing identically on $\gamma_{m} \backslash \Gamma_{\ell n}$, since the zero extension operator is bounded from $H_{00}^{1 / 2}$ to $H_{00}^{1 / 2}$ we get

$$
\left\|\left[\bar{w}_{\delta}-u\right]\right\|_{H_{00}^{1 / 2}\left(\gamma_{m}\right)} \lesssim \sum_{n \in I_{\ell}^{(i)}}\left\|\zeta^{n}\right\|_{H_{00}^{1 / 2}\left(\gamma_{m}\right)} \lesssim \sum_{n \in I_{\ell}^{(i)}}\left\|\left[\bar{w}_{\delta}-u\right]\right\|_{H_{00}^{1 / 2}\left(\Gamma_{\ell n}\right)}
$$

whence, since on $\Gamma_{\ell n},\left|\left[\bar{w}_{\delta}-u\right]\right| \leq\left|\Pi_{\delta, \ell} u-u\right|+\left|\Pi_{\delta, n} u-u\right|$

$$
\sum_{m \in I}\left\|\pi_{m}\left(\left[\bar{w}_{\delta}-u\right]\right)\right\|_{H_{00}^{1 / 2}\left(\gamma_{m}\right)} \lesssim \sum_{\ell, n}\left\|\Pi_{\delta, \ell} u-u\right\|_{H_{00}^{1 / 2}\left(\Gamma_{\ell n}\right)} .
$$

The conclusion follows by observing that

$$
\left\|\Pi_{\delta, \ell} u-u\right\|_{H_{00}^{1 / 2}\left(\Gamma_{\ell n}\right)} \lesssim \delta_{\ell}^{t_{\ell}-1}\|u\|_{t_{\ell}, \Omega_{\ell}} .
$$

This last bound can be proven by space interpolation [35]. On one hand, we have $\Pi_{\delta, \ell} u-u \in H_{0}^{\bar{s}-1 / 2}\left(\Gamma_{\ell n}\right)$ and

$$
\begin{aligned}
\left\|\Pi_{\delta, \ell} u-u\right\|_{\bar{s}-1 / 2, \Gamma_{\ell n}} & \lesssim\left\|\Pi_{\delta, \ell} u-u\right\|_{\bar{s}-1 / 2, \partial \Omega_{\ell}} \\
& \lesssim\left\|\Pi_{\delta, \ell} u-u\right\|_{\bar{s}, \Omega_{\ell}} \lesssim \delta_{\ell}^{t_{\ell}-\bar{s}}\|u\|_{t_{\ell}, \Omega_{\ell}} .
\end{aligned}
$$

On the other hand

$$
\left\|\Pi_{\delta, \ell} u-u\right\|_{1 / 4, \Gamma_{\ell n}} \lesssim\left\|\Pi_{\delta, \ell} u-u\right\|_{3 / 4, \Omega_{\ell}} \lesssim \delta_{\ell}^{t_{\ell}-3 / 4}\|u\|_{t_{\ell}, \Omega_{\ell}} .
$$

Since $H_{00}^{1 / 2}\left(\Gamma_{\ell n}\right)$ can be obtained as the interpolated of order $\theta=1 /[4(\bar{s}-1)+1]$ between $H^{1 / 4}\left(\Gamma_{\ell n}\right)$ and $H_{0}^{\bar{s}-1 / 2}\left(\Gamma_{\ell n}\right)$, we obtain that

$$
\left\|\Pi_{\delta, \ell} u-u\right\|_{H_{00}^{1 / 2}\left(\Gamma_{\ell n}\right)} \lesssim \delta_{\ell}^{(1-\theta)\left(t_{\ell}-3 / 4\right)} \delta_{\ell}^{\theta\left(t_{\ell}-\bar{s}\right)}\|u\|_{t_{\ell}, \Omega_{\ell}}=\delta_{\ell}^{t_{\ell}-1}\|u\|_{t_{\ell}, \Omega_{\ell}} .
$$

Corollary 2.18. Under the assumptions of Theorem 2.16, if for all $\ell=1, \ldots, L$ the solution $u$ of (10) satisfies $\left.u\right|_{\Omega_{\ell}} \in H^{t_{\ell}}\left(\Omega_{\ell}\right)$ for some $t_{\ell}, \bar{s} \leq t_{\ell} \leq \bar{t}$ then

$$
\left\|u-u_{\delta}\right\|_{X} \lesssim \inf _{\lambda \in M_{\delta}}\left\|\frac{\partial u}{\partial \nu}-\lambda\right\|_{-1 / 2, S}+\sum_{\ell=1}^{L} \delta_{\ell}^{t_{\ell}-1}\|u\|_{t_{\ell}, \Omega_{\ell}} .
$$


Remark 2.19. If we consider the Lagrange multiplier formulation of problem (29), Corollary 2.12 would imply that a discrete inf-sup condition of the form

$$
\inf _{\lambda_{\delta} \in M_{\delta}} \sup _{u_{\delta} \in X_{\delta}} \frac{\int_{S}\left[u_{\delta}\right] \lambda_{\delta}}{\left\|\lambda_{\delta}\right\|_{-1 / 2, S}\left\|u_{\delta}\right\|_{X}} \geq \alpha(h)>0
$$

is fulfilled with a stability constant $\alpha=\alpha(h)$, decreasing as $|\log h|^{-1}$. In fact, it is easy to see that Fortin's Lemma [13] can be applied by setting the Fortin projector equals to $(I d-\pi)$. An alternative to such an approach is to work with suitable mesh dependent norms. Then, in the finite element framework, a discrete inf-sup condition can be proven to hold uniformly in $h$ [12]. However, working with mesh dependent norms usually yields (when, as in the present framework it is not possible to work "triangle by triangle"), a dependence of the constant in the estimates obtained on the ratio between the coarser and the finer "mesh sizes" of each subdomain. This is due to the concurrent use of direct and inverse inequalities. In the case of a very non uniform discretization, as one would have in an adaptive wavelet scheme, such a ratio can be much larger than the logarithmic factor in the bound (44) and in the resulting error estimate. Therefore, though for the sake of simplicity we will concentrate, later on, on a uniform wavelet discretization, we chose here to use natural norms such as $\|\cdot\|_{-1 / 2, S}$. We remark that in the geometrically conforming case, if the solution is sufficiently regular, we still get an optimal error estimate (see Th. 2.16 and 3.21).

\section{THE MORTAR WAVELET METHOD}

We now come to the problem of constructing mortar approximation spaces in a general wavelet context. In view of Remark 2.15 we focus here on one (rectangular) subdomain $\Omega_{\ell}$ which for simplicity we identify with the unit square. The approximation spaces on $] 0,1\left[^{2}\right.$ will be obtained from tensor-product of one-dimensional spaces. Starting from a (now classical) multiresolution analysis on the interval (see for instance [2, 19]), we construct a wavelet family (adapted to the "multiplier" sides) which will allow to define a suitable multiplier space verifying by construction the basic assumptions (A.1-A.3) needed to apply the abstract result of the previous section.

\subsection{Multiresolution analyses on the interval and approximation properties}

\subsubsection{Scaling functions on the interval}

The construction of multiresolution analyses and associated wavelet bases on the interval, which preserve the approximation properties of the wavelet bases on $\mathbb{R}$ has nowadays already been discussed in a number of papers (see $[2,19]$ for the first constructions, but also $[18,25,30,34])$. To be as general as possible, we will consider the case of biorthogonal wavelet bases, that includes the one of orthonormal bases. Let us point out which are the properties of such bases, which will be needed for the design and the analysis of the mortar wavelet method and which we will assume to be verified by the chosen basis. We recall that a wide class of bases exists, which satisfy by construction such assumptions.

We assume that we are given a couple of biorthogonal multi-resolution analyses (MRA) of $L^{2}(0,1)$, that is a couple of increasing sequences of finite dimensional subspaces $\left(V_{j}=V_{j}(] 0,1[)\right)_{j \geq j_{0}}$ and $\left(\tilde{V}_{j}=\tilde{V}_{j}(] 0,1[)\right)_{j \geq j_{0}}$, whose respective union is dense in $L^{2}(0,1)$. Without loss of generality, we can assume that $\operatorname{dim} V_{j}=\operatorname{dim} \widetilde{V}_{j}=$ $2^{j}+2$ (see $[2,21,32]$ for example).

The spaces $V_{j}$ and $\tilde{V}_{j}$ are respectively spanned by biorthogonal scaling function Riesz bases $\left(\varphi_{j, k}\right)_{k=0, \ldots, 2^{j}+1}$ and $\left(\tilde{\varphi}_{j, k}\right)_{k=0, \ldots, 2^{j}+1}$, verifying:

$$
\int_{0}^{1} \varphi_{j, k} \tilde{\varphi}_{j, k}=\delta_{k k^{\prime}}, \forall k, k^{\prime}=0, \ldots, 2^{j}+1 .
$$


We recall that by the definition of Riesz's basis, the two following norm equivalences hold uniformly in $j$ :

$$
\left\|\sum_{k=0}^{2^{j}+1} u_{k} \varphi_{j, k}\right\|_{0,] 0,1[} \simeq\left(\sum_{k=0}^{2^{j}+1}\left|u_{k}\right|^{2}\right)^{1 / 2}
$$

and

$$
\left\|\sum_{k=0}^{2^{j}+1} u_{k} \tilde{\varphi}_{j, k}\right\|_{0,] 0,1[} \simeq\left(\sum_{k=0}^{2^{j}+1}\left|u_{k}\right|^{2}\right)^{1 / 2} .
$$

The scaling function bases are usually constructed as a modification of the corresponding (compactly supported) scaling functions in $L^{2}(\mathbb{R})$ (see [27]). In this process, one naturally distinguishes between edge (left and right) functions and interior functions: interior functions coincide with scaling functions on the line whose support is included into ]0,1[ while edge function are linear combinations of scaling functions on the line (restricted to ]0,1[), whose support overlaps the left (resp. right) edge. Consequently, for $j \geq j_{0}$, the scaling basis of $V_{j}$ is usually indexed as follows, with $N$ a given integer:

- $\varphi_{j, k}^{\text {left }}, k=0, \ldots, N-1$, the $N$ scaling functions at the left edge;

- $\varphi_{j, k}, k=N, \ldots, 2^{j}-N+1$, the interior scaling functions;

- $\varphi_{j, k}^{r i g h t}, k=2^{j}-N+2, \ldots, 2^{j}+1$, the $N$ scaling functions at the right edge.

Similarly for the scaling basis of $\tilde{V}_{j}$ biorthogonal (again $\tilde{N}$ is a given integer):

- $\tilde{\varphi}_{j, k}^{l e f t}, k=0, \ldots, \tilde{N}-1$, the $\tilde{N}$ scaling functions at the left edge;

- $\tilde{\varphi}_{j, k}, k=\tilde{N}, \ldots, 2^{j}-\tilde{N}+1$, the interior scaling functions;

- $\tilde{\varphi}_{j, k}^{r i g h t}, k=2^{j}-\tilde{N}+2, \ldots, 2^{j}+1$, the $\tilde{N}$ scaling functions at the right edge.

For notational simplicity, we will omit in the following the suffixes "left" and "right". We will then denote $\left(\varphi_{j, k}\right)_{k=0, \ldots, 2^{j}+1}$ and $\left(\tilde{\varphi}_{j, k}\right)_{k=0, \ldots, 2^{j}+1}$ the above bases. We recall that these functions have compact support and are scale invariant, i.e. $\forall j \geq j_{0}, \forall x \in[0,1]$, and $\forall k=0, \ldots, N-1$ (resp. $\forall k=0, \ldots, \tilde{N}-1$ for the second equality) on the left boundary it holds

$$
\varphi_{j, k}(x)=2^{\frac{j-j_{0}}{2}} \varphi_{j_{0}, k}\left(2^{j-j_{0}} x\right), \quad \tilde{\varphi}_{j, k}(x)=2^{\frac{j-j_{0}}{2}} \tilde{\varphi}_{j_{0}, k}\left(2^{j-j_{0}} x\right),
$$

while on the right boundary we have $\forall k=2^{j}-N+2, \ldots, 2^{j}+1\left(\right.$ resp. $\left.\forall k=2^{j}-\tilde{N}+2, \ldots, 2^{j}+1\right)$

$$
\begin{aligned}
& \varphi_{j, k}(1-x)=2^{\frac{j-j_{0}}{2}} \varphi_{j_{0}, k}\left(2^{j-j_{0}}(1-x)\right), \\
& \tilde{\varphi}_{j, k}(1-x)=2^{\frac{j-j_{0}}{2}} \tilde{\varphi}_{j_{0}, k}\left(2^{j-j_{0}}(1-x)\right) .
\end{aligned}
$$

Moreover, the interior scaling functions coincide with the original scaling functions on the real line and $\forall j \geq j_{0}$, $\forall k=N, \ldots, 2^{j}-N+1$, and they take the form:

$$
\varphi_{j, k}(x)=\varphi_{j, N}\left(x-2^{-j}(k-N)\right)=2^{j / 2} \varphi\left(2^{j} x-k\right),
$$

where $\varphi(x)=2^{-j_{0} / 2} \varphi_{j_{0}, N}\left(2^{-j_{0}}(x+N)\right)$ is the scaling function of the corresponding multiscale analysis for $L^{2}(\mathbb{R})$. An analogous relation holds for the duals $\tilde{\varphi}_{j, k}$.

Finally we can also suppose, that all scaling functions of $V_{j}$ vanish at the edges 0 and 1 , except one function at each edge. For example we will assume that only the functions $\varphi_{j, 0}$ and $\varphi_{j, 2^{j}+1}$ verify a non-vanishing boundary 
condition:

$$
\begin{aligned}
& \varphi_{j, 0}(0) \neq 0 \quad \text { and } \quad \varphi_{j, 2^{j}+1}(1) \neq 0, \\
& \varphi_{j, k}(0)=0, \quad \forall k=1, \ldots, 2^{j}+1 \text {, } \\
& \varphi_{j, k}(1)=0, \quad \forall k=0, \ldots, 2^{j} .
\end{aligned}
$$

We will assume that the two $M R A$ are respectively $R$-regular and $\tilde{R}$-regular, that is for all $j, k$ in the range considered it holds $\varphi_{j, k} \in H^{R}(0,1)$ and $\tilde{\varphi}_{j, k} \in H^{\tilde{R}}(0,1)$, with $R \geq 1, \tilde{R}>0$.

Following [19], these scaling functions are constructed in such a way that they satisfy Strang-Fix conditions, that is they allow to reconstruct polynomials up to degree $N-1$ in the space $V_{j}$ and up to degree $\tilde{N}-1$ in the space $\tilde{V}_{j}$. More precisely, we will have for all $j \geq j_{0}$ and for $n=0, \ldots, N-1$

$$
2^{j / 2}\left(2^{j} x\right)^{n}=\sum_{k=0}^{2^{j}+1} a_{k}^{n} \varphi_{j, k}(x), \text { and } 2^{j / 2}\left(2^{j}(1-x)\right)^{n}=\sum_{k=0}^{2^{j}+1} b_{k}^{n} \varphi_{j, k}(x)
$$

and for $n=0, \ldots, \tilde{N}-1$

$$
2^{j / 2}\left(2^{j} x\right)^{n}=\sum_{k=0}^{2^{j}+1} \tilde{a}_{k}^{n} \tilde{\varphi}_{j, k}(x), \text { and } 2^{j / 2}\left(2^{j}(1-x)\right)^{n}=\sum_{k=0}^{2^{j}+1} \tilde{b}_{k}^{n} \tilde{\varphi}_{j, k}(x),
$$

with $a_{k}^{n}$ and $\tilde{a}_{k}^{n}$ reals independent of $j$. We recall that the parameters $R, \tilde{R}, N$ and $\tilde{N}$ necessarily satisfy the relations $R \leq N$ and $\tilde{R} \leq \tilde{N}$.

Let now $\bar{V}_{j}^{0} \subset H_{0}^{1}(0, \overline{1})$ be the space of functions of $V_{j}$ vanishing at the boundaries of the interval:

$$
\begin{gathered}
V_{j}^{0}=V_{j} \cap H_{0}^{1}(0,1)=\operatorname{span}\left\langle\varphi_{j, k}, k=1, \ldots, 2^{j}\right\rangle, \\
V_{j}=V_{j}^{0} \oplus \operatorname{span}\left\langle\varphi_{j, 0}, \varphi_{j, 2^{j}+1} .\right\rangle
\end{gathered}
$$

When designing the mortar wavelet method, the space $V_{j}^{0}$ will play the role of the space which, in Section 2.3, was denoted $T_{m}^{0}$, that is the space of traces on a "multiplier side" of discrete functions, vanishing at the extrema. The corresponding multiplier space (which will play the role of the $M_{\delta}^{m}$ of Sect. 2.3) is most naturally defined with the aid of a suitable dual space, which will have to satisfy the assumptions of Section 2.5. The construction of such dual space for $V_{j}^{0}$ is the object of the following theorem.

Theorem 3.1. Let $j \geq j_{0}$. Assume that the two linear systems

$$
\sum_{k=0}^{\tilde{N}-1} \tilde{a}_{k}^{n} \alpha_{k}=\tilde{a}_{\tilde{N}}^{n}, \text { and } \sum_{k=2^{j}-\tilde{N}+2}^{2^{j}+1} \tilde{b}_{k}^{n} \beta_{k}=\tilde{b}_{2^{j}-\tilde{N}+1}^{n}, \forall n=0, \tilde{N}-1
$$


admit solutions $\left(\alpha_{k}\right)_{k=0, \tilde{N}-1}$ and $\left(\beta_{k}\right)_{k=2^{j}-\tilde{N}+2,2^{j}+1}$ satisfying $\alpha_{0} \neq 0$ and $\beta_{2^{j}+1} \neq 0$. Then the family:

$$
\begin{array}{lll}
\tilde{\varphi}_{j, k}^{*} & =\tilde{\varphi}_{j, k}-\frac{\alpha_{k}}{\alpha_{0}} \tilde{\varphi}_{j, 0}, & \\
\tilde{\varphi}_{j, \tilde{N}}^{*} & =\tilde{\varphi}_{j, \tilde{N}}+\frac{1}{\alpha_{0}} \tilde{\varphi}_{j, 0}, & \\
\tilde{\varphi}_{j, k}^{*} & =\tilde{\varphi}_{j, k}, & \text { for } k=\tilde{N}+1,2^{j}-\tilde{N}-1 \\
\tilde{\varphi}_{j, 2^{j}-\tilde{N}+1}^{*} & =\tilde{\varphi}_{j, 2^{j}-\tilde{N}+1}+\frac{1}{\beta_{2^{j}+1}} \tilde{\varphi}_{j, 2^{j}+1}, & \\
\tilde{\varphi}_{j, k}^{*} & =\tilde{\varphi}_{j, k}-\frac{\beta_{k}}{\beta_{2^{j}+1}} \tilde{\varphi}_{j, 2^{j}+1}, & \text { for } k=2^{j}-\tilde{N}+2,2^{j}
\end{array}
$$

is biorthogonal to the basis $\left\{\varphi_{j, k}, k=1, \ldots, 2^{j}\right\}$ of $V_{j}^{0}$, that is for all $n, k=1, \ldots, 2^{j}$ it holds

$$
\int_{0}^{1} \varphi_{j, k} \tilde{\varphi}_{j, n}^{*}=\delta_{n, k}
$$

Moreover the space $\tilde{V}_{j}^{*}$ defined by:

$$
\tilde{V}_{j}^{*}=\operatorname{span}\left\langle\tilde{\varphi}_{j, k}^{*}, k=1, \ldots, 2^{j}\right\rangle
$$

contains all polynomials of degree $\tilde{N}-1$.

Remark that $\tilde{V}_{j}^{*}$ does not verify homogeneous boundary conditions.

Proof. The biorthogonality between families $\left(\tilde{\varphi}_{j, k}^{*}\right)$ and $\left(\varphi_{j, k}\right)$ is a trivial consequence of the biorthogonality between $\left(\tilde{\varphi}_{j, k}\right)$ and $\left(\varphi_{j, k}\right)$. By construction, the $\left(\tilde{\varphi}_{j, k}\right)$ reproduce polynomials up to order $\tilde{N}-1$. Indeed, by equation (58) we have, $\forall n=0, \ldots, \tilde{N}-1$ :

$$
\begin{aligned}
2^{j / 2}\left(2^{j} x\right)^{n} & =\sum_{k=0}^{2^{j}+1} \tilde{a}_{k}^{n} \tilde{\varphi}_{j, k}(x) \\
& =\left(\tilde{a}_{0}^{n}+\sum_{k=1}^{\tilde{N}-1} \tilde{a}_{k}^{n} \frac{\alpha_{k}}{\alpha_{0}}-\frac{\tilde{a}_{\tilde{N}}^{n}}{\alpha_{0}}\right) \tilde{\varphi}_{j, 0}(x)+\sum_{k=1}^{2^{j}-\tilde{N}} \tilde{a}_{k}^{n} \tilde{\varphi}_{j, k}^{*}(x)+\sum_{k=2^{j}-\tilde{N}+1}^{2^{j}+1} \tilde{a}_{k}^{n} \tilde{\varphi}_{j, k}(x)
\end{aligned}
$$

where we applied the definition (60).

Since the $\alpha_{k}$ satisfy the following equation:

$$
\tilde{a}_{\tilde{N}}^{n}-\sum_{k=0}^{\tilde{N}-1} \tilde{a}_{k}^{n} \alpha_{k}=0, \forall n=0, \tilde{N}-1
$$

we have

$$
2^{j / 2}\left(2^{j} x\right)^{n}=\sum_{k=1}^{2^{j}-\tilde{N}} \tilde{a}_{k}^{n} \tilde{\varphi}_{j, k}^{*}(x)+\sum_{k=2^{j}-\tilde{N}+1}^{2^{j}+1} \tilde{a}_{k}^{n} \tilde{\varphi}_{j, k}(x) .
$$


Then each polynomial $P$ of degree $\tilde{N}$ writes:

$$
P(x)=\sum_{k=1}^{2^{j}-\tilde{N}} p_{k} \tilde{\varphi}_{j, k}^{*}(x)+\sum_{k=2^{j}-\tilde{N}+1}^{2^{j}+1} p_{k} \tilde{\varphi}_{j, k}(x)
$$

with $p_{k}=<P \mid \varphi_{j, k}>$ for $k=1, \ldots, 2^{j}+1$. In particular

$$
2^{j / 2}\left(2^{j}(1-x)\right)^{n}=\sum_{k=1}^{2^{j}-\tilde{N}} \tilde{b}_{k}^{n} \tilde{\varphi}_{j, k}^{*}(x)+\sum_{k=2^{j}-\tilde{N}+1}^{2^{j}+1} \tilde{b}_{k}^{n} \tilde{\varphi}_{j, k}(x) .
$$

Using (60) again this yields

$$
2^{j / 2}\left(2^{j}(1-x)\right)^{n}=\sum_{k=1}^{2^{j}-\tilde{N}} \tilde{b}_{k}^{n} \tilde{\varphi}_{j, k}^{*}(x)+\left(\tilde{b}_{2^{j}-\tilde{N}+1}^{n}+\sum_{k=2^{j}-N+2}^{2^{j}} \tilde{b}_{k}^{n} \frac{\beta_{k}}{\beta_{2^{j}+1}}-\frac{\tilde{b}_{2^{j}-\tilde{N}+1}^{n}}{\beta_{2^{j}+1}}\right) \tilde{\varphi}_{j, 2^{j}+1}(x) .
$$

Since the $\beta_{k}$ satisfy the following equation:

$$
\tilde{b}_{2^{j}-\tilde{N}+1}^{n}-\sum_{k=2^{j}-N+2}^{2^{j}+1} \tilde{b}_{k}^{n} \beta_{k}=0, \forall n=0, \tilde{N}-1
$$

we have

$$
2^{j / 2}\left(2^{j}(1-x)\right)^{n}=\sum_{k=1}^{2^{j}} \tilde{b}_{k}^{n} \tilde{\varphi}_{j, k}^{*}(x) .
$$

Since clearly the set $\left\{2^{j / 2}\left(2^{j}(1-x)\right)^{n}, n=0, \ldots, \tilde{N}-1\right\}$ generates the set of polynomials of degrees less or equal than $\tilde{N}-1$, this yields the thesis.

Remark 3.2. We assumed here that the matrices $\left[\tilde{a}_{k}^{n}\right]_{0 \leq k, n \leq \tilde{N}-1}$ and $\left[\tilde{b}_{k}^{n}\right]_{2^{j}+\tilde{N}+2 \leq k, n \leq 2^{j}+1}$ are invertible and that the coefficients $\alpha_{0}$ and $\beta_{0}$ are non vanishing. This can be proven in particular cases, such as for biorthogonal spline wavelets $[20,25]$ or orthogonal wavelets. In this last example $\varphi_{j, k}=\tilde{\varphi}_{j, k}$ and for instance on the left boundary the coefficients $\tilde{a}_{k}^{n}=\int_{0}^{1} x^{n} \tilde{\varphi}_{j, k}$ can be written as $\tilde{a}_{k}^{n}=p_{n}(k)$, where $p_{n}$ is a polynomial of degree $n$ [32]. $\left[\tilde{a}_{k}^{n}\right]_{0 \leq k, n \leq \tilde{N}-1}$ is then a nonsingular Vandermonde type matrix. The fact that the coefficients $\alpha_{0}$ and $\beta_{2^{j}+1}$ are non vanishing descends from the same argument, by considering the matrix $\left[\tilde{a}_{k}^{n}\right]_{0 \leq n \leq \tilde{N}-1,1 \leq k \leq \tilde{N}}$. In general we will have to verify case by case that such an assumption holds. Remark that, thanks to the scale invariance property of the scaling functions, it will be enough to verify it once and for all for $j=j_{0}$.

\subsubsection{Projectors on MRA spaces and approximation properties}

Let $P_{j}$ and $\tilde{P}_{j}$ be the biorthogonal projectors associated to $V_{j}$ and $\tilde{V}_{j}$ defined, as usual, for all $\eta$ and $\lambda$ in $L^{2}(0,1)$ by:

$$
\begin{aligned}
P_{j}(\eta) & =\sum_{k=0}^{2^{j}+1}\left\langle\eta \mid \tilde{\varphi}_{j, k}\right\rangle \varphi_{j, k} \\
\tilde{P}_{j}(\lambda) & =\sum_{k=0}^{2^{j}+1}\left\langle\lambda \mid \varphi_{j, k}\right\rangle \tilde{\varphi}_{j, k}
\end{aligned}
$$


where $\langle\cdot \mid \cdot\rangle$ denotes the $L^{2}(0,1)$ scalar product.

It is well known that under the assumptions of the previous section, the following Jackson and Bernstein inequalities hold:

Theorem 3.3. Let $0 \leq r \leq s \leq N, r \leq R$, then for all $\eta \in H^{s}(0,1)$, we have:

$$
\left\|\eta-P_{j}(\eta)\right\|_{r,] 0,1[} \lesssim 2^{-j(s-r)}\|\eta\|_{s,] 0,1[},
$$

while, for $0 \leq r \leq s \leq \tilde{N}, r \leq \tilde{R}$ and for all $\lambda \in H^{s}(0,1)$ it holds:

$$
\left\|\lambda-\tilde{P}_{j}(\lambda)\right\|_{r,] 0,1[} \lesssim 2^{-j(s-r)}\|\lambda\|_{s,] 0,1[} .
$$

Moreover, a scaling argument yields the following inverse inequalities

Theorem 3.4. For all $r, s, 0 \leq r \leq s \leq R$ one has:

$$
\|\eta\|_{s,] 0,1[} \lesssim 2^{j(s-r)}\|\eta\|_{r,] 0,1[}, \text { if } \eta \in V_{j}
$$

and for all $r, s, 0 \leq r \leq s \leq \tilde{R}$ one has:

$$
\|\lambda\|_{s,] 0,1[} \lesssim 2^{j(s-r)}\|\lambda\|_{r,] 0,1[}, \text { if } \lambda \in \tilde{V}_{j} .
$$

In the following it will also be useful to consider the biorthogonal projector on $V_{j}^{0}$ induced by its dual $\tilde{V}_{j}^{*}$ and its adjoint $\tilde{\pi}_{j}$.

Definition 3.5. Let $\pi_{j}: L^{2}(0,1) \rightarrow V_{j}^{0}$ be defined, for all $\eta \in L^{2}(0,1)$ by:

$$
\pi_{j}(\eta)=\sum_{k=1}^{2^{j}}\left\langle\eta \mid \tilde{\varphi}_{j, k}^{*}\right\rangle \varphi_{j, k} .
$$

Moreover let $\tilde{\pi}_{j}: L^{2}(0,1) \rightarrow \tilde{V}_{j}^{*}$ be the adjoint of $\pi_{j}$ :

$$
\tilde{\pi}_{j}(\lambda)=\sum_{k=1}^{2^{j}}\left\langle\lambda \mid \varphi_{j, k}\right\rangle \tilde{\varphi}_{j, k}^{*} .
$$

The natural environment for analyzing the projectors $\pi_{j}$ consists in the spaces with null zero-th order trace. More precisely, for $s \geq 1$ we denote by $\mathcal{H}_{0}^{s}(0,1)$ the space

$$
\mathcal{H}_{0}^{s}(0,1)=H^{s}(0,1) \cap H_{0}^{1}(0,1),
$$

endowed with the $H^{s}$ norm. For $s=0$ we set $\mathcal{H}_{0}^{0}(0,1)=L^{2}(0,1)$, and for $0<s<1$ we define $\mathcal{H}_{0}^{s}$ by space interpolation. We remark that $\mathcal{H}_{0}^{1 / 2}(0,1)=H_{00}^{1 / 2}(0,1)$.

Since $V_{j}^{0} \subset V_{j}$ and $\tilde{V}_{j}^{*} \subset \tilde{V}_{j}$ and since they reproduce locally respectively all polynomials of degree $N-1$ satisfying homogeneous boundary condition at the edge 0 and 1 and polynomials of degree $\tilde{N}-1$, the following bounds also hold.

Theorem 3.6. For all $r \leq R$, for all $s, 0 \leq r \leq s \leq N, \pi_{j}$ is continuous from $\mathcal{H}_{0}^{s}(0,1)$ to $\mathcal{H}_{0}^{r}(0,1)$, and for all $\eta \in \mathcal{H}_{0}^{s}(0,1)$ we have:

$$
\left\|\eta-\pi_{j}(\eta)\right\|_{\mathcal{H}_{0}^{r}(0,1)} \lesssim 2^{-j(s-r)}\|\eta\|_{\mathcal{H}_{0}^{s}(0,1)}
$$


For all $r \leq \tilde{R}$, for all $s, 0 \leq r \leq s \leq \tilde{N}$, for all $\lambda \in H^{s}(0,1)$, we have:

$$
\left\|\lambda-\tilde{\pi}_{j}(\lambda)\right\|_{r,] 0,1[} \lesssim 2^{-j(s-r)}\|\lambda\|_{s,] 0,1[}
$$

Moreover, for all $0 \leq r \leq s \leq R$, for all $\eta \in V_{j}^{0}$, it holds

$$
\|\eta\|_{\mathcal{H}_{0}^{s}(0,1)} \lesssim 2^{j(s-r)}\|\eta\|_{\mathcal{H}_{0}^{r}(0,1)}
$$

Proof. It is not difficult to check that both $\pi_{j}$ and $\tilde{\pi}_{j}$ are $L^{2}(0,1)$ bounded projectors. Let us for example consider $\pi_{j}(\eta)$ : using the definition of $\varphi_{j, k}^{*}$ and the Riesz's basis property (54) it is not difficult to realize that

$$
\left\|\pi_{j}(\eta)\right\|_{0,] 0,1[}^{2}=\left\|\sum_{k=1}^{2^{j}}\left\langle\eta \mid \tilde{\varphi}_{j, k}^{*}\right\rangle \varphi_{j, k}\right\|_{0,] 0,1[}^{2} \lesssim \sum_{k=0}^{2^{j}+1}\left|\left\langle\eta \mid \tilde{\varphi}_{j, k}\right\rangle\right|^{2} \lesssim\left\|P_{j}(\eta)\right\|_{0,] 0,1[}^{2} \lesssim\|\eta\|_{0,] 0,1[}^{2}
$$

As far as $\tilde{\pi}_{j}$ is concerned, we first observe that, by the definition of $\tilde{\varphi}_{j, k}^{*}$ and using the Riesz's basis property (54) it holds

$$
\left\|\sum_{k=1}^{2^{j}} u_{k} \tilde{\varphi}_{j, k}^{*}\right\|_{0,] 0,1[}^{2} \lesssim \sum_{k=1}^{2^{j}}\left|u_{k}\right|^{2}+\left|\sum_{k=1}^{\tilde{N}} c_{k} u_{k}\right|^{2}+\left|\sum_{k=2^{j}-\tilde{N}}^{2^{j}} d_{k} u_{k}\right|^{2} \lesssim \sum_{k=1}^{2^{j}}\left|u_{k}\right|^{2}
$$

where the coefficients $c_{k}$ and $d_{k}$ are given by (60):

$$
c_{k}=-\frac{\alpha_{k}}{\alpha_{0}}, k=1, \ldots, \tilde{N}, \quad c_{0}=\frac{1}{\alpha_{0}}, \quad d_{k}=-\frac{\beta_{k}}{\beta_{2^{j}+1}}, k=2^{j}-\tilde{N}+2, \ldots, 2^{j}, \quad d_{2^{j}-\tilde{N}+1}=\frac{1}{\beta_{2^{j}+1}}
$$

Then

$$
\left\|\tilde{\pi}_{j}(\lambda)\right\|_{0,] 0,1[}^{2}=\left\|\sum_{k=1}^{2^{j}}\left\langle\lambda \mid \varphi_{j, k}\right\rangle \tilde{\varphi}_{j, k}^{*}\right\|_{0,] 0,1[}^{2} \lesssim \sum_{k=1}^{2^{j}}\left|\left\langle\lambda \mid \varphi_{j, k}\right\rangle\right|^{2} \lesssim\left\|\tilde{P}_{j}(\lambda)\right\|_{0,] 0,1[}^{2} \lesssim\|\lambda\|_{0,] 0,1[}^{2}
$$

Using the polynomial reproduction properties of the spaces $V_{j}^{0}$ and $\tilde{V}_{j}^{*}$, as well as the Bernstein inequalities (69) and (70), the theorem then results by applying standard arguments.

In particular it holds

Corollary 3.7. $\pi_{j}$ is continuous from $H_{00}^{1 / 2}(0,1)$ to $H_{00}^{1 / 2}(0,1)$, that is for all $\eta \in H_{00}^{1 / 2}(0,1)$ :

$$
\left\|\pi_{j}(\eta)\right\|_{H_{00}^{1 / 2}(0,1)} \lesssim\|\eta\|_{H_{00}^{1 / 2}(0,1)}
$$

Moreover, for all $\eta \in L^{2}(0,1)$ and for all $\lambda \in \tilde{V}_{j}^{*}$,

$$
\int_{0}^{1}\left(\eta-\pi_{j}(\eta)\right) \lambda=0
$$

\subsection{Wavelets}

We can now introduce two couples of biorthogonal wavelet bases, which will both be needed for the analysis and/or for the implementation of the mortar wavelet method. On one hand we will need the complement spaces $W_{j}$ and $\tilde{W}_{j}$ defined as follows. 
Definition 3.8. The complement spaces $W_{j}$ and its dual $\tilde{W}_{j}$ are defined by:

$$
\begin{gathered}
W_{j}=\left(P_{j+1}-P_{j}\right) V_{j+1}, \\
\tilde{W}_{j}=\left(\tilde{P}_{j+1}-\tilde{P}_{j}\right) \tilde{V}_{j+1} .
\end{gathered}
$$

Let us now introduce the complement space $W_{j}^{0}$ of $V_{j}^{0}$ in $V_{j+1}^{0}$ and its dual. To do this, we will use the biorthogonal projector $\tilde{\pi}_{j}$ defined in the previous section.

Definition 3.9. The complement spaces $W_{j}^{0}$ and its dual $\tilde{W}_{j}^{*}$ are defined by:

$$
\begin{aligned}
& W_{j}^{0}=\left(\pi_{j+1}-\pi_{j}\right) V_{j+1}^{0}, \\
& \tilde{W}_{j}^{*}=\left(\tilde{\pi}_{j+1}-\tilde{\pi}_{j}\right) \tilde{V}_{j+1}^{*} .
\end{aligned}
$$

Following [20] it is possible to construct wavelet bases for $W_{j}, \tilde{W}_{j}, W_{j}^{0}$ and $\tilde{W}_{j}^{*}$. We will then have biorthogonal wavelet Riesz bases $\left\{\psi_{j, k}, k=1, \cdots, 2^{j}\right\},\left\{\tilde{\psi}_{j, k}, k=1, \cdots, 2^{j}\right\}$, and $\left\{\psi_{j, k}^{0}, k=1, \cdots, 2^{j}\right\},\left\{\tilde{\psi}_{j, k}^{*}, k=1, \cdots, 2^{j}\right\}$ such that the following identities hold for all $f \in L^{2}(0,1)$ :

$$
\begin{aligned}
\left(P_{j+1}-P_{j}\right) f & =\sum_{k=1}^{2^{j}}\left\langle f \mid \tilde{\psi}_{j, k}\right\rangle \psi_{j, k}, \\
\left(\tilde{P}_{j+1}-\tilde{P}_{j}\right) f & =\sum_{k=1}^{2^{j}}\left\langle f \mid \psi_{j, k}\right\rangle \tilde{\psi}_{j, k}, \\
\left(\pi_{j+1}-\pi_{j}\right) f & =\sum_{k=1}^{2^{j}}\left\langle f \mid \tilde{\psi}_{j, k}^{*}\right\rangle \psi_{j, k}^{0}, \\
\left(\tilde{\pi}_{j+1}-\tilde{\pi}_{j}\right) f & =\sum_{k=1}^{2^{j}}\left\langle f \mid \psi_{j, k}^{0}\right\rangle \tilde{\psi}_{j, k}^{*} .
\end{aligned}
$$

Remark 3.10. The functions in the complement spaces $\tilde{W}_{j}^{*}$ do not satisfy an homogeneous boundary condition.

As usual in the wavelet framework, norm equivalences hold for Sobolev spaces of negative and/or fractional smoothness (either of the type $H^{s}(0,1)$ or $\mathcal{H}_{0}^{s}(0,1)$ as defined above), in terms the expansions in either one of the bases $\left\{\psi_{j, k}\right\},\left\{\tilde{\psi}_{j, k}\right\},\left\{\psi_{j, k}^{0}\right\}$ and $\left\{\tilde{\psi}_{j, k}^{*}\right\}$. It is beyond the goals of this paper to precisely state such norm equivalences. We will therefore limit ourselves to state the following theorem, which will play a key role in both the analysis and the implementation of the Mortar Wavelet Method and which can be proven by applying the results of $[14,23]$.

Theorem 3.11. Let $f \in L^{2}(0,1)$. The following two norm equivalences hold:

$$
\begin{array}{ll}
\forall f \in H^{1 / 2}(0,1), \quad\|f\|_{1 / 2, j 0,1]}^{2} & \sim \sum_{k=0}^{2^{j_{0}}+1}\left|\left\langle f \mid \tilde{\varphi}_{j_{0}, k}\right\rangle\right|^{2}+\sum_{j \geq j_{0}} \sum_{k=1}^{2^{j}} 2^{j}\left|\left\langle f \mid \tilde{\psi}_{j, k}\right\rangle\right|^{2}, \\
\forall f \in H_{00}^{1 / 2}(0,1), \quad\|f\|_{H_{00}^{1 / 2}(0,1)}^{2} & \sim \sum_{k=1}^{2^{j_{0}}}\left|\left\langle f \mid \tilde{\varphi}_{j_{0}, k}^{*}\right\rangle\right|^{2}+\sum_{j \geq j_{0}} \sum_{k=1}^{2^{j}} 2^{j}\left|\left\langle f \mid \tilde{\psi}_{j, k}^{*}\right\rangle\right|^{2} .
\end{array}
$$

Remark 3.12. Moreover $f \in H^{1 / 2}(0,1)$ (resp. $\left.f \in H_{00}^{1 / 2}(0,1)\right)$ if and only if the sum on the right hand side of (84) (resp. (85)) is finite. 


\subsection{Mortar approximation spaces in the wavelet framework}

\subsubsection{D multiresolution analyses}

As usual in the unit $] 0,1\left[^{2}\right.$ we will consider as approximation spaces a two-dimensional $M R A \mathcal{V}_{j}$ defined by tensor products of one-dimensional $M R A$ :

$$
\begin{aligned}
& \mathcal{V}_{j}=V_{j} \otimes V_{j} \sim \operatorname{span}\left\langle\Phi_{j, \mathbf{k}}, \mathbf{k} \in \mathbb{K}_{j}\right\rangle \\
& \tilde{\mathcal{V}}_{j}=\tilde{V}_{j} \otimes \tilde{V}_{j} \sim \operatorname{span}\left\langle\tilde{\Phi}_{j, \mathbf{k}}, \mathbf{k} \in \mathbb{K}_{j}\right\rangle
\end{aligned}
$$

where the multi-index set $\mathbb{K}_{j}$ is defined by $\mathbb{K}_{j}=\left\{\mathbf{k}=\left(k_{1}, k_{2}\right), k_{1}, k_{2}=0, \ldots, 2^{j}+1\right\}=\left\{0, \ldots, 2^{j}+1\right\}^{2}$ and where, using the notation $(f \otimes g)(x, y)=f(x) g(y)$ the functions $\Phi_{j, \mathbf{k}}$ are defined by

$$
\Phi_{j, \mathbf{k}}=\varphi_{j, k_{1}} \otimes \varphi_{j, k_{2}} \quad \text { and } \quad \tilde{\Phi}_{j, \mathbf{k}}=\tilde{\varphi}_{j, k_{1}} \otimes \tilde{\varphi}_{j, k_{2}}
$$

In the same way, $\mathcal{V}_{j}^{0}$, the subspaces of $\mathcal{V}_{j}$ verifying homogeneous boundary conditions and its dual $\tilde{\mathcal{V}}_{j}^{*}$ are, for $\mathbb{K}_{j}^{*}=\left\{1, \ldots, 2^{j}\right\}^{2} \subset \mathbb{K}_{j}$

$$
\begin{aligned}
& \mathcal{V}_{j}^{0}=V_{j}^{0} \otimes V_{j}^{0} \sim \operatorname{span}\left\langle\Phi_{j, \mathbf{k}}, \mathbf{k} \in \mathbb{K}_{j}^{*}\right\rangle, \\
& \tilde{\mathcal{V}}_{j}^{*}=\tilde{V}_{j}^{*} \otimes \tilde{V}_{j}^{*} \sim \operatorname{span}\left\langle\tilde{\Phi}_{j, \mathbf{k}}^{*}, \mathbf{k} \in \mathbb{K}_{j}^{*}\right\rangle .
\end{aligned}
$$

It is well known that the family $\left(\mathcal{V}_{j}\right)$ constitutes a $M R A$ of $L^{2}(] 0,1\left[^{2}\right)$, and $\left(\mathcal{V}_{j}^{0}\right)$ a $M R A$ of $H_{0}^{1}(] 0,1\left[^{2}\right)$. The two-dimensional biorthogonal projections on respectively $\mathcal{V}_{j}$ and $\mathcal{V}_{j}^{0}$ will be denoted by $\mathcal{P}_{j}$ and $\Pi_{j}$ and their adjoint by $\tilde{\mathcal{P}}_{j}$ and $\tilde{\Pi}_{j}$. They are defined respectively as:

$$
\begin{array}{ll}
\mathcal{P}_{j} f=\sum_{\mathbf{k} \in \mathbb{K}_{j}}\left\langle f \mid \tilde{\Phi}_{j, \mathbf{k}}\right\rangle \Phi_{j, \mathbf{k}}, & \Pi_{j} f=\sum_{\mathbf{k} \in \mathbb{K}_{j}^{*}}\left\langle f \mid \tilde{\Phi}_{j, \mathbf{k}}^{*}\right\rangle \Phi_{j, \mathbf{k}}, \\
\tilde{\mathcal{P}}_{j} f=\sum_{\mathbf{k} \in \mathbb{K}_{j}}\left\langle f \mid \Phi_{j, \mathbf{k}}\right\rangle \tilde{\Phi}_{j, \mathbf{k}}, & \tilde{\Pi}_{j} f=\sum_{\mathbf{k} \in \mathbb{K}_{j}^{*}}\left\langle f \mid \Phi_{j, \mathbf{k}}\right\rangle \tilde{\Phi}_{j, \mathbf{k}}^{*} .
\end{array}
$$

Two-dimensional wavelets are constructed (as usual) by tensor products of univariate bases. In particular, we will then have two couples of biorthogonal wavelet bases, which we will denote by $\left\{\Psi_{j, \kappa}\right\}_{\kappa \in \mathbb{I}_{j}},\left\{\tilde{\Psi}_{j, \kappa}\right\}_{\kappa \in \mathbb{I}_{j}}$, and $\left\{\Psi_{j, \kappa}^{0}\right\}_{\kappa \in \mathbb{I}_{j}^{*}},\left\{\tilde{\Psi}_{j, \kappa}^{*}\right\}_{\kappa \in \mathbb{I}_{j}^{*}}$ defined in such a way that:

$$
\begin{aligned}
& \left(\mathcal{P}_{j+1}-\mathcal{P}_{j}\right) f=\sum_{\kappa \in \mathbb{I}_{j}}\left\langle f \mid \tilde{\Psi}_{j, \kappa}\right\rangle \Psi_{j, \kappa} \\
& \left(\tilde{\mathcal{P}}_{j+1}-\tilde{\mathcal{P}}_{j}\right) f=\sum_{\kappa \in \mathbb{I}_{j}}\left\langle f \mid \Psi_{j, \kappa}\right\rangle \tilde{\Psi}_{j, \kappa} \\
& \left(\Pi_{j+1}-\Pi_{j}\right) f=\sum_{\kappa \in \mathbb{I}_{j}^{*}}\left\langle f \mid \tilde{\Psi}_{j, \kappa}^{*}\right\rangle \Psi_{j, \kappa}^{0} \\
& \left(\tilde{\Pi}_{j+1}-\tilde{\Pi}_{j}\right) f=\sum_{\kappa \in \mathbb{I}_{j}^{*}}\left\langle f \mid \Psi_{j, \kappa}^{0}\right\rangle \tilde{\Psi}_{j, \kappa}^{*},
\end{aligned}
$$


where $\mathbb{I}_{j}$ and $\mathbb{I}_{j}^{*}$ will denote suitable multi-index sets. The corresponding complement spaces will then be denoted by

$$
\begin{aligned}
& \mathcal{W}_{j}=\left(\mathcal{P}_{j+1}-\mathcal{P}_{j}\right) \mathcal{V}_{j}=\operatorname{span}\left\langle\Psi_{j, \kappa}, \quad \kappa \in \mathbb{I}_{j}\right\rangle \\
& \tilde{\mathcal{W}}_{j}=\left(\tilde{\mathcal{P}}_{j+1}-\tilde{\mathcal{P}}_{j}\right) \mathcal{V}_{j}=\operatorname{span}\left\langle\tilde{\Psi}_{j, \kappa}, \kappa \in \mathbb{I}_{j}\right\rangle \\
& \mathcal{W}_{j}^{0}=\left(\Pi_{j+1}-\Pi_{j}\right) \mathcal{V}_{j}=\operatorname{span}\left\langle\Psi_{j, \kappa}^{0}, \kappa \in \mathbb{I}_{j}^{*}\right\rangle \\
& \tilde{\mathcal{W}}_{j}^{*}=\left(\tilde{\Pi}_{j+1}-\tilde{\Pi}_{j}\right) \mathcal{V}_{j}=\operatorname{span}\left\langle\tilde{\Psi}_{j, \kappa}^{*}, \kappa \in \mathbb{I}_{j}^{*}\right\rangle
\end{aligned}
$$

Remark 3.13. There are several ways of building wavelet bases for the spaces $\mathcal{W}_{j}, \tilde{\mathcal{W}}_{j}, \mathcal{W}_{j}^{0}$ and $\tilde{\mathcal{W}}_{j}^{*}$. The basis which is classically used in the context of wavelet discretization is constructed starting from the observation that the space $\mathcal{W}_{j}$ can be decomposed as

$$
\mathcal{W}_{j}=\left(V_{j} \otimes W_{j}\right) \oplus\left(W_{j} \otimes V_{j}\right) \oplus\left(W_{j} \otimes W_{j}\right) .
$$

The functions in the basis take then the three forms $\varphi_{j, k} \otimes \psi_{j, k^{\prime}}, \psi_{j, k} \otimes \varphi_{j, k^{\prime}}$ and $\psi_{j, k} \otimes \psi_{j, k^{\prime}}$.

A second basis for $\mathcal{W}_{j}$ can be obtained by further decomposing $V_{j}$ as $V_{j}=V_{j_{0}} \oplus_{m=j_{0}}^{j-1} W_{m}$, which yields a decomposition for $\mathcal{W}_{j}$ of the form

$$
\mathcal{W}_{j}=\left(V_{j_{0}} \otimes W_{j}\right) \oplus\left(W_{j} \otimes V_{j_{0}}\right) \oplus_{m=j_{0}}^{j-1}\left(W_{m} \otimes W_{j}\right) \oplus_{m=j_{0}}^{j}\left(W_{j} \otimes W_{m}\right)
$$

The basis functions take then the forms $\varphi_{j_{0}, n} \otimes \psi_{j, k}, \psi_{j, k} \otimes \varphi_{j_{0}, n}, \psi_{m_{2}, n} \otimes \psi_{j, k}$ and $\psi_{j, k} \otimes \psi_{m, n}$ (with $m \leq j$ ). The same alternative holds for the three other spaces $\tilde{\mathcal{W}}_{j}, \mathcal{W}_{j}^{0}$ and $\tilde{\mathcal{W}}_{j}^{*}$. Depending on the choice made, the index sets $\mathbb{I}_{j}$ and $\mathbb{I}_{j}^{*}$ will have different forms. In the first case, the multi-index $\kappa$ will be a triplet $\kappa=\left(k, k^{\prime}, \epsilon\right)$, where the "type parameter" $\epsilon=1,2,3$ distinguishes between the three forms $\varphi_{j, k} \otimes \psi_{j, k^{\prime}}, \psi_{j, k} \otimes \varphi_{j, k^{\prime}}$ and $\psi_{j, k} \otimes \psi_{j, k^{\prime}}$. In the second case, it will be a quadruplet $\left(m, n, m^{\prime}, n^{\prime}\right)$ with suitable restrictions on the values of $m$ and $m^{\prime}\left(\max \left\{m, m^{\prime}\right\}=j\right.$ and, using the convention $\left.\psi_{j_{0}-1, k}=\varphi_{j_{0}, k}, m, m^{\prime} \geq j_{0}-1\right)$. We remark however that Theorem 3.15 in the following holds for both choices.

Again, Jackson and Bernstein inequalities and norm equivalences analogous to (67-73) and (84-85) are still valid in dimension 2. In particular, the following two theorems hold.

Theorem 3.14. For all $r, 0 \leq r \leq R$, for all $s, r \leq s \leq N$ and for all $u \in H^{s}(] 0,1\left[^{2}\right)$ it holds

$$
\left\|u-\mathcal{P}_{j}(u)\right\|_{r,] 0,1[2} \lesssim 2^{-j(s-r)}\|u\|_{s,] 0,1[2 .} .
$$

For all $r, s, 0 \leq r \leq s \leq R$, and for all $u \in \mathcal{V}_{j}$

$$
\|u\|_{s,] 0,1\left[^{2}\right.} \lesssim 2^{j(s-r)}\|u\|_{r,] 0,1\left[^{2}\right.}
$$

Theorem 3.15. The following norm equivalences hold: for all $u \in H^{1}(] 0,1\left[{ }^{2}\right)$

$$
\|u\|_{1,] 0,1\left[^{2}\right.}^{2} \sim \sum_{\mathbf{k} \in \mathbb{K}_{j_{0}}}\left|\left\langle u \mid \tilde{\Phi}_{j_{0}, \mathbf{k}}\right\rangle\right|^{2}+\sum_{j \geq j_{0}} \sum_{\kappa \in \mathbb{I}_{j}} 2^{2 j}\left|\left\langle u \mid \tilde{\Psi}_{j, \kappa}\right\rangle\right|^{2},
$$

and for all $u \in H_{0}^{1}(] 0,1\left[^{2}\right)$

$$
\|u\|_{1,] 0,1\left[^{2}\right.}^{2} \sim \sum_{\mathbf{k} \in \mathbb{K}_{j_{0}}^{*}}\left|\left\langle u \mid \tilde{\Phi}_{j_{0}, \mathbf{k}}^{*}\right\rangle\right|^{2}+\sum_{j \geq j_{0}} \sum_{\kappa \in \mathbb{I}_{j}^{*}} 2^{2 j}\left|\left\langle u \mid \tilde{\Psi}_{j, \kappa}^{*}\right\rangle\right|^{2} .
$$




\subsubsection{Trace and multiplier spaces}

In order to use such spaces in the framework of the mortar method, for each edge of $] 0,1\left[^{2}\right.$ we need to define a space suitably coupled with the space of traces of the functions in $\mathcal{V}_{j}$, as required in Section 2.5 , to be used as multiplier space if the edge is chosen to be a multiplier edge.

Let then $\gamma$ be any edge of $] 0,1\left[2\right.$. Thanks to the tensor product structure of the space $\mathcal{V}_{j}$, the space $T_{j}(\gamma)$ of traces of functions of $\mathcal{V}_{j}$ can be identified to the space spanned by the basis of scaling functions on the interval:

$$
T_{j}(\gamma)=\left.\mathcal{V}_{j}\right|_{\gamma} \sim V_{j}=\operatorname{span}\left\langle\varphi_{j, k}, k=0, \ldots, 2^{j}+1\right\rangle
$$

Then, the space $T_{j}^{0}(\gamma) \subset H_{00}^{1 / 2}(\gamma)$ of functions of $T_{j}(\gamma)$ vanishing at the extrema of $\gamma$ verifies:

$$
T_{j}^{0}(\gamma) \sim V_{j}^{0}=\operatorname{span}\left\langle\varphi_{j, k}, k=1, \ldots, 2^{j}\right\rangle
$$

The choice of the multiplier space on the edge $\gamma$ is the object of the following definition.

Definition 3.16. If $\gamma$ is a multiplier side a natural choice for the multiplier space $M_{j}(\gamma)$ on $\gamma$ will be:

$$
M_{j}(\gamma) \sim \tilde{V}_{j}^{*}=\operatorname{span}\left\langle\tilde{\varphi}_{j, k}^{*}, k=1, \ldots, 2^{j}\right\rangle
$$

Remark 3.17. The multiplier space $M_{j}$ verifies $M_{j} \subset \tilde{T}_{j}$, and not $M_{j} \subset T_{j}$ as usual in the mortar methods, see [8] (we fall back in the classical mortar method framework, i.e. $M_{j}$ is a subspace of codimension 2 of $T_{j}$, if orthonormal wavelets are considered). Such a choice has some advantage over a choice implying $M_{j} \subset T_{j}$. Such a space is in fact exactly the dual space of $T_{j}^{0}$. Thanks to the biorthogonality property of the two bases for the spaces $M_{j}$ and $T_{j}^{0}$, the matrix appearing in the linear system that has to be solved for computing the projector $\pi_{j}$ is diagonal. This also happens for spectral approximation, while for P1 finite elements, the corresponding linear system involves a tridiagonal matrix. We recall that the computation of the projector $\pi_{j}$ is needed for imposing the constraint in the numerical resolution of the linear system stemming from (29), if one wants to avoid the explicit construction of a basis for $\mathcal{X}_{\delta}$.

Theorem 3.18. The spaces $T_{j}^{0}(\gamma)$ and $M_{j}(\gamma)$ verify assumptions (A.1-A.3).

Proof. The projection: $\pi_{j}: L^{2}(\gamma) \rightarrow T_{j}^{0}(\gamma)$ defined by (71) verifies assumption (A1). This result is given by Corollary 3.7. Moreover, if $\gamma$ is a multiplier side of $] 0,1\left[{ }^{2}\right.$, for all $\eta \in T_{j}^{0}$ and $s<\frac{1}{2}$, we have by (75):

$$
\|\eta\|_{H_{00}^{1 / 2}(\gamma)} \lesssim 2^{-j(s-1 / 2)}\|\eta\|_{s, \gamma}
$$

and the assumption (A.3) is fulfilled with $h=2^{-j}$. Then we only need to prove that (A2) holds.

For simplicity let us assume that $\gamma=\{(x, 0), x \in] 0,1[\}$. The following map: $R_{j}: T_{j}^{0}(\gamma) \rightarrow \mathcal{V}_{j}$ defined, for all $\eta \in T_{j}^{0}(\gamma)$ by:

$$
R_{j}(\eta)=\sum_{j^{\prime}=j_{0}}^{j-1} \sum_{k=1}^{2^{j^{\prime}}} \frac{\left\langle\eta \mid \tilde{\psi}_{j^{\prime}, k}^{*}\right\rangle}{\varphi_{j^{\prime}, 0}(0)} \psi_{j^{\prime}, k}^{0} \otimes \varphi_{j^{\prime}, 0}+\sum_{k=1}^{2^{j_{0}}} \frac{\left\langle\eta \mid \tilde{\varphi}_{j_{0}, k}^{*}\right\rangle}{\varphi_{j_{0}, 0}(0)} \varphi_{j_{0}, k} \otimes \varphi_{j_{0}, 0}
$$

is a lifting from $H_{00}^{1 / 2}(\gamma)$ to $H^{1}(] 0,1\left[^{2}\right)$ which verifies assumption (A2).

In fact, let us consider the $M R A \check{\mathcal{V}}_{j}=V_{j}^{0} \otimes V_{j}$ and its dual $\hat{\mathcal{V}}_{j}=\tilde{V}_{j}^{*} \otimes \tilde{V}_{j}$. If we denote by $\left\{\check{\Psi}_{j, \kappa}, \kappa \in \check{\mathbb{I}}_{j}\right\}$ and $\left\{\hat{\Psi}_{j, \kappa}, \kappa \in \check{\mathbb{I}}_{j}\right\}$, the corresponding couple of biorthogonal wavelet bases, (with an analogous notation for scaling functions) the following norm equivalence holds: for all $u \in H_{0}^{1}(] 0,1\left[^{2}\right)$, satisfying $u(0, y) \equiv u(1, y) \equiv 0$ for all 
$y \in] 0,1[:$

$$
\|u\|_{1, j 0,1\left[^{2}\right.}^{2} \sim \sum_{\mathbf{k} \in \tilde{\mathbb{K}}_{j_{0}}}\left|\left\langle u \mid \tilde{\Phi}_{j_{0}, \kappa}\right\rangle\right|^{2}+\sum_{j \geq j_{0}} 2^{2 j} \sum_{\kappa \in \tilde{\mathbb{I}}_{j}}\left|\left\langle f \mid \hat{\Psi}_{j, \kappa}\right\rangle\right|^{2}
$$

where $\check{K}_{j_{0}}=\left\{1, \ldots, 2^{j_{0}}\right\} \times\left\{0, \ldots, 2^{j_{0}}+1\right\}$. Now we observe that for suitable $\kappa=\kappa(k)$ we have

$$
\check{\Psi}_{j, \kappa}(x, y)=\psi_{j, k}^{0}(x) \varphi_{j, 0}(y), \quad \hat{\Psi}_{j, \kappa}(x, y)=\tilde{\psi}_{j, k}^{*}(x) \tilde{\varphi}_{j, 0}(y) .
$$

Therefore, applying the previous norm equivalence to $R_{j}(\eta)$, and observing that $\varphi_{j, 0}(0) \simeq 2^{j / 2}$ we get

$$
\left\|R_{j}(\eta)\right\|_{1,] 0,1\left[^{2}\right.} \sim \sum_{k=1}^{2^{j_{0}}} 2^{-j_{0}}\left|\left\langle\eta \mid \tilde{\varphi}_{j_{0}, k}^{*}\right\rangle\right|^{2}+\sum_{j^{\prime}=j_{0}}^{j-1} \sum_{k=1}^{2^{j^{\prime}}} 2^{j^{\prime}}\left|\left\langle\eta \mid \tilde{\psi}_{j^{\prime}, k}^{*}\right\rangle\right|^{2},
$$

and the right hand side of (98) is equivalent to $\|\eta\|_{H_{00}^{1 / 2}(\gamma)}$, thanks to norm equivalence (85).

\subsection{Error estimates}

We can now use the function spaces just defined in the framework of the mortar method described in Section 2. For simplicity we will just consider the case in which all the subdomains are discretized by wavelets. More precisely, for each $\ell=1, \ldots, L$ let $\left.F_{\ell}: \Omega_{\ell} \rightarrow\right] 0,1\left[{ }^{2}\right.$ be a linear mapping of the (rectangular) subdomain $\Omega_{\ell}$ onto the reference domain and for each edge $\gamma_{\ell}^{(i)}$ let $\left.F_{\ell}^{(i)}: \gamma_{\ell}^{(i)} \rightarrow\right] 0,1\left[\left(\left.F_{\ell}^{(i)} \sim F_{\ell}\right|_{\gamma_{\ell}^{(i)}}\right)\right.$ be the mapping induced on $\gamma_{\ell}^{(i)}$ by $F_{\ell}$.

In each subdomain $\Omega_{\ell}$ let the approximation space $\mathcal{V}_{\delta}^{\ell}$ be given by:

$$
\mathcal{V}_{\delta}^{\ell}=\mathcal{V}_{j(\ell)} \circ F_{\ell}
$$

with some fixed $j(\ell) \geq j_{0}$. To each edge $\gamma_{\ell}^{(i)}$ we associate the trace space of $\mathcal{V}_{\delta}^{\ell}$ :

$$
T_{\ell, i}=T_{j(\ell)} \circ F_{\ell}^{(i)}
$$

For $m=(\ell, i) \in I\left(\gamma_{m}=\gamma_{\ell}^{(i)}\right.$ multiplier side $)$, the multiplier space is

$$
M_{\delta}^{m}=M_{j(\ell)} \circ F_{\ell}^{(i)} .
$$

The constrained approximation space $\mathcal{X}_{\delta}$ and its multiplier space $M_{\delta}$ are then defined according to $(27,26)$.

If the decomposition is geometrically conforming, the resulting multiplier space $M_{\delta}$ will automatically satisfy assumption (31) (ensuring stability), while in the case of a geometrically non conforming decomposition we will have to choose $j(\ell)$ in such a way that either one of the sufficient conditions I and II of Section 2.4 are satisfied. This is true, provided one of the following two conditions is satisfied (we recall that $I_{\ell}^{(i)}$ is defined as the set of those subdomains whose boundary intersect $\gamma_{\ell}^{(i)}$ (see (5)):

(i): $\operatorname{card}\left\{I_{\ell}^{(i)}\right\} \leq \tilde{N}$ ( $\tilde{N}$ being, we recall, the number of polynomials exactly reproduced in $\tilde{V}_{j_{0}}$ ); in this case stability will hold for all $j \geq j_{0}$;

(ii): if (i) does not hold, then we need that for each $n \in I_{\ell}^{(i)}$ there exists $\tilde{\varphi}_{j, k}^{*} \in M_{\delta}^{m}$ such that $\operatorname{supp} \tilde{\varphi}_{j, k}^{*} \subset \Gamma_{\ell n}$; this certainly holds provided that

$$
j(\ell) \geq \max _{n \in I_{\ell}^{(i)}} \inf \left\{j: \exists \tilde{\varphi}_{j, n}^{*} \in M_{j}\left(\gamma_{m}\right), \operatorname{supp} \tilde{\varphi}_{j, n}^{*} \subset \Gamma_{\ell n}\right\} .
$$


With such a choice of the spaces, we will consider problem (29). Since assumptions (A.1-A.3) are fulfilled (as we checked in the previous section), the solution $u_{\delta}$ of such problem will satisfy the following error estimate.

Corollary 3.19. Let $u$ be solution of (10), and let $u_{\delta}$ be the solution of problem (PD-29), with the above choice of approximation and multiplier spaces. If $\left.u\right|_{\Omega_{\ell}} \in H^{s_{\ell}}\left(\Omega_{\ell}\right), s_{\ell}>3 / 2$, then it holds

$$
\begin{aligned}
\left\|u-u_{\delta}\right\|_{X} \lesssim & (1+|\log h|)\left(\sum_{\ell=1}^{L} 2^{-2 j(\ell)\left(s_{\ell}-1\right)}\|u\|_{s_{\ell}, \Omega_{\ell}}^{2}\right)^{1 / 2}+ \\
& +\left(\sum_{m=(\ell, i) \in I} 2^{-2 j(\ell)\left(s_{\ell}-1\right)}\left\|\frac{\partial u}{\partial \nu}\right\|_{s_{\ell}-3 / 2, \gamma_{m}}^{2}\right)^{1 / 2}
\end{aligned}
$$

Proof. Either one of the conditions (i) and (ii) is sufficient to ensure that assumption (BP) is satisfied with a constant $\bar{C}$ ultimately depending on the geometric decomposition of $\Omega$ in subdomains and on the particular wavelet space chosen. Since the approximation and multiplier spaces satisfy assumption (A.1-A.3), we can then apply Corollary 2.14, which, together with the Jackson type inequalities (91) and (74) yields (100).

Remark 3.20. In the case of a geometrically non conforming decomposition, condition (ii) might be quite restrictive, forcing the discretization to be very fine $\left(2^{-j(\ell)} \leq N^{-1}\right.$ inf $\left.\left|\Gamma_{\ell n}\right|\right)$. It is opinion of the authors that such condition can in practice be relaxed by only asking $2^{-j(\ell)} \leq \inf \left|\Gamma_{\ell n}\right|$.

In the geometrically conforming case we have instead the following (optimal) error estimate:

Theorem 3.21. If the decomposition is geometrically conforming, under the assumptions of Corollary 3.19, it holds

$$
\left\|u-u_{\delta}\right\|_{X} \lesssim\left(\sum_{\ell=1}^{L} 2^{-2 j(\ell)\left(s_{\ell}-1\right)}\|u\|_{s_{\ell}, \Omega_{\ell}}^{2}\right)^{1 / 2}+\left(\sum_{m=(\ell, i) \in I} 2^{-2 j(\ell)\left(s_{\ell}-1\right)}\left\|\frac{\partial u}{\partial \nu}\right\|_{s_{\ell}-3 / 2, \gamma_{m}}^{2}\right)^{1 / 2}
$$

Proof. The result follows from Corollary 2.18, provided it exits a projector $\check{\Pi}_{j}$ satisfying the assumptions of Theorem 2.16. Indeed, observing that for a geometrically conforming decomposition all cross points are vertices of all the subdomains to which they belong, the projector $\check{\Pi}_{j}: H^{s}(] 0,1\left[^{2}\right) \rightarrow \mathcal{V}_{j}$ can be defined as:

$$
\check{\Pi}_{j}(u)=\mathcal{P}_{j}(u)+\sum_{i=1}^{4}\left(\frac{u\left(A_{i}\right)}{\Phi_{j}^{A_{i}}\left(A_{i}\right)}-\left\langle u \mid \tilde{\Phi}_{j}^{A_{i}}\right\rangle\right) \Phi_{j}^{A_{i}}
$$

where for each vertex $A_{i}$ of $[0,1]^{2}$ we use the notation $\Phi_{j}^{A_{i}}$ to indicate the only scaling function among the elements of the basis of $\mathcal{V}_{j}$ that does not vanish at $A_{i}$, (that is for instance:

$$
\begin{aligned}
& \text { if } A_{0}=(0,0), \text { then } \quad \Phi_{j}^{A_{0}}(x, y)=\varphi_{j, 0}(x) \varphi_{j, 0}(y), \\
& \text { if } A_{1}=(1,0), \text { then } \quad \Phi_{j}^{A_{1}}(x, y)=\varphi_{j, 2^{j}+1}(x) \varphi_{j, 0}(y),
\end{aligned}
$$

and so on). It is easily verified that $\check{\Pi}_{j}$ is indeed a projector and that it verifies $\check{\Pi}_{j}(u)\left(A_{i}\right)=u\left(A_{i}\right)$ for any vertex $A_{i}$. Moreover it is not difficult to check that the Jackson inequality of the form (48) is valid for $\bar{s}=R$ and $\bar{t}=N$, provided $\check{\Pi}_{j}$ is bounded from $H^{t}\left(\Omega_{\ell}\right)$ to $H^{s}\left(\Omega_{\ell}\right)$. This is the case if $1<t$. 


\subsection{Some remarks on the implementation}

It is well known that in the implementation of the mortar method, the multiplication by the stiffness matrix can be performed by applying subdomainwise the local stiffness matrix (which does not take into account the constraints), after multiplication by a "transfer matrix" which gives the values of the constrained degrees of freedom (the ones living on the interior of multiplier sides) in terms of the remaining (free) degrees of freedom. The transfer matrix is a discrete realization of the projector $\pi$ (Sect. 2.5).

In the case that a geometrically conforming decomposition is considered and that all subdomains are discretized by wavelets, applying such projector $\left(\pi_{j}\right.$, see $\left.(71)\right)$ reduces to performing either a fast wavelet transform or an inverse fast wavelet transform depending on which of the two discretizations on the "trace" and on the "multiplier" side is finer.

In general, when a non geometrically conforming decomposition is considered and/or wavelets are coupled with some other method, in the case in which a multiplier side $\gamma_{m}$ is chosen in a subdomain discretized by wavelets, then, thanks to (71), applying the transfer matrix reduces to computing the scalar products of the functions on the corresponding "trace sides" with the scaling functions $\tilde{\varphi}_{j, k}^{*}, k=1, \ldots, 2^{j}$. We refer to [16] for an analysis of the effect that using numerical quadrature in computing the projector $\pi$ has on the method.

Furthermore, in the numerical resolution of the linear system arising from the wavelet mortar method it is possible to take advantage of the features of wavelet basis in order to design efficient preconditioners . This can be done for instance by using the good spectral properties of wavelets in an iterative substructuring approach [10].

\section{REFERENCES}

[1] Y. Achdou, G. Abdoulaev, Y. Kutznetsov and C. Prud'homme, On the parallel inplementation of the mortar element method. ESAIM: M2AN 33 (1999) 245-259.

[2] L. Anderson, N. Hall, B. Jawerth and G. Peters, Wavelets on closed subsets on the real line, in Topics in the theory and applications of wavelets, L.L. Schumaker and G. Webb, Eds., Academic Press, Boston (1993) 1-61.

[3] F. Ben Belgacem, The mortar finite element method with Lagrange multiplier. Numer. Math. 84 (1999) 173-197.

[4] F. Ben Belgacem, A. Buffa and Y. Maday, The mortar element method for 3D Maxwell's equations. C. R. Acad. Sci. Paris Sér. I Math. 329 (1999) 903-908.

[5] F. Ben Belgacem and Y. Maday, Non conforming spectral method for second order elliptic problems in 3D. East-West J. Numer. Math. 4 (1994) 235-251.

[6] C. Bernardi, Y. Maday, C. Mavripilis and A.T. Patera, The mortar element method applied to spectral discretizations, in Finite element analysis in fluids. Proc. of the seventh international conference on finite element methods in flow problems, T. Chung and G. Karr, Eds., UAH Press (1989).

[7] C. Bernardi, Y. Maday and A.T. Patera, Domain decomposition by the mortar element method, in Asymptotic and numerical methods for partial differential equations with critical parameters, H.G. Kaper and M. Garbey, Eds., N.A.T.O. ASI Ser. C 384 .

[8] C. Bernardi, Y. Maday and A.T. Patera, A new nonconforming approach to domain decomposition: the mortar element method, in Nonlinear partial differential equations and their applications, Collège de France Seminar XI, H. Brezis and J.L.Lions, Eds. (1994) 13-51.

[9] S. Bertoluzza, An adaptive wavelet collocation method based on interpolating wavelets, in Multiscale wavelet methods for partial differential equations. W. Dahmen, A.J. Kurdila and P. Oswald, Eds., Academic Press 6 (1997) 109-135.

[10] S. Bertoluzza and V. Perrier, The mortar method in the wavelet context. Technical Report 99-17, LAGA, Université Paris 13 (1999).

[11] S. Bertoluzza and P. Pietra, Space frequency adaptive approximation for quantum hydrodynamic models. Transport Theory Statist. Phys. 28 (2000) 375-395.

[12] D. Braess and W. Dahmen, Stability estimate of the mortar finite element method for 3-dimensional problems. East-West J. Numer. Math. 6 (1998) 249-264.

[13] F. Brezzi and M. Fortin, Mixed and hybrid finite element methods. Springer-Verlag, New York (1991).

[14] C. Canuto and A. Tabacco, Multilevel decomposition of functional spaces. J. Fourier Anal. Appl. 3 (1997) 715-742.

[15] C. Canuto, A. Tabacco and K. Urban, The wavelet element method. Part I: Construction and analysis. Appl. Comput. Harmon. Anal. ACHA 6 (1999) 1-52.

[16] L. Cazabeau, C. Lacour and Y. Maday, Numerical quadratures and mortar methods, in Computational Sciences for the 21st Century, Bristeau et al., Eds., John Wiley \& Sons, New York (1997) 119-128.

[17] P. Charton and V. Perrier, A pseudo-wavelet scheme for the two-dimensional Navier-Stokes equation. Comput. Appl. Math. 15 (1996) 139-160. 
[18] G. Chiavassa and J. Liandrat, On the effective construction of compactly supported wavelets satisfying homogeneous boundary conditions on the interval. Appl. Comput. Harmon. Anal. ACHA 4 (1997) 62-73.

[19] A. Cohen, I. Daubechies and P. Vial, Wavelets on the interval and fast wavelet transforms. Appl. Comput. Harmon. Anal. ACHA 1 (1993) 54-81.

[20] A. Cohen and R. Masson, Wavelet methods for second order elliptic problems, preconditioning and adaptivity. SIAM J. Sci. Comput. 21 (1999) 1006-1026.

[21] A. Cohen and R. Masson, Wavelet adaptive method for second order elliptic problems. boundary conditions and domain decomposition. Numer. Math. 86 (1999) 193-238.

[22] S. Dahlke, W. Dahmen ans R. Hochmut and R. Schneider, Stable multiscale bases and local error estimation for elliptic problems. Appl. Numer. Math. 23 (1997) 21-48.

[23] W. Dahmen, Stability of multiscale transformations. J. Fourier Anal. Appl. 2 (1996) 341-361.

[24] W. Dahmen and A. Kunoth, Multilevel preconditioning. Numer. Math. 63 (1992) 315-344.

[25] W. Dahmen, A. Kunoth and K. Urban, Biorthogonal spline-wavelets on the interval - stability and moment condition. Appl. Comput. Harmon. Anal. ACHA 6 (1999) 132-196.

[26] W. Dahmen and R. Schneider, Composite wavelet bases for operator equations. Math. Comp. 68 (1999) 1533-1567.

[27] I. Daubechies, Ten lectures on wavelets, in CBMS-NSF Regional Conference Series in Applied Mathematics 61. SIAM, Philadelphia (1992).

[28] S. Jaffard, Wavelet methods for fast resolution of elliptic problems. SIAM J. Numer. Anal. 29 (1992) 965-986.

[29] Y. Maday, V. Perrier and J.C. Ravel, Adaptivité dynamique sur bases d'ondelettes pour l'approximation d'équations aux dérivées partielles. C. R. Acad. Sci. Paris Sér. I Math. 312 (1991) 405-410.

[30] R. Masson, Biorthogonal spline wavelets on the interval for the resolution of boundary problems. $M^{3} A S$ (Math. Models Methods Appl. Sci.) 6 (1996) 749-791.

[31] Y. Meyer, Ondelettes et opérateurs. Hermann, Paris (1990).

[32] P. Monasse and V. Perrier, Orthonormal wavelet bases adapted for partial differential equations with boundary conditions. SIAM J. Math. Anal. 29 (1998) 1040-1065.

[33] C. Prud'homme, A strategy for the resolution of the tridimensional incompressible Navier-Stokes equations, in Méthodes itératives de décomposition de domaines et communications en calcul parallèle. Calcul. Parallèles Réseaux Syst. Répartis 10 Hermès (1998) 371-380.

[34] S. Grivet Talocia and A. Tabacco, Wavelets on the interval with optimal localization. $M^{3}$ AS (Math. Models Methods Appl. Sci.) 10 (2000) 441-462.

[35] H. Triebel, Interpolation theory, function spaces, differential operators. North Holland-Elsevier Science Publishers, Amsterdam (1978).

[36] B. Wohlmut, A mortar finite element method using dual spaces for the Lagrange multiplier. SIAM J. Numer. Anal. 38 (2000) 989-1012.

To access this journal online:

www.edpsciences.org 